\title{
Nearby supernova host galaxies from the CALIFA Survey
}

\section{Sample, data analysis, and correlation to star-forming regions ${ }^{\star}$}

\author{
L. Galbany ${ }^{1,2,3, \star \star}$, V. Stanishev ${ }^{1}$, A. M. Mourão ${ }^{1}$, M. Rodrigues ${ }^{4,5}$, H. Flores $^{5}$, R. García-Benito ${ }^{6}$, D. Mast ${ }^{7}$, \\ M. A. Mendoza ${ }^{6}$, S. F. Sánchez ${ }^{8}$, C. Badenes ${ }^{9}$, J. Barrera-Ballesteros ${ }^{10,11}$, J. Bland-Hawthorn ${ }^{12}$, J. Falcón-Barroso ${ }^{10,11}$, \\ B. García-Lorenzo ${ }^{10,11}$, J. M. Gomes ${ }^{13}$, R. M. González Delgado ${ }^{6}$, C. Kehrig ${ }^{6}$, M. Lyubenova ${ }^{14}$, \\ A. R. López-Sánchez ${ }^{15,16}$, A. de Lorenzo-Cáceres ${ }^{17}$, R. A. Marino ${ }^{18}$, S. Meidt ${ }^{13}$, M. Mollá ${ }^{19}$, P. Papaderos ${ }^{13}$, \\ M. A. Pérez-Torres ${ }^{6,20,21}$, F. F. Rosales-Ortega ${ }^{22}$, G. van de Ven ${ }^{14}$, and the CALIFA Collaboration
}

(Affiliations can be found after the references)

Received 30 July 2014 / Accepted 4 September 2014

\begin{abstract}
We use optical integral field spectroscopy (IFS) of nearby supernova (SN) host galaxies $(0.005<z<0.03)$ provided by the Calar Alto Legacy Integral Field Area (CALIFA) Survey with the goal of finding correlations in the environmental parameters at the location of different SN types. In this first study of a series we focus on the properties related with star formation (SF). We recover the sequence in association of different $\mathrm{SN}$ types to the star-forming regions by using several indicators of the ongoing and recent SF related to both the ionized gas and the stellar populations. While the total ongoing SF is on average the same for the three SN types, SNe Ibc/Ilb tend to occur closer to star-forming regions and in higher SF density locations than SNe II and SNe Ia; the latter shows the weakest correlation. SNe Ia host galaxies have masses that are on average 0.3-0.8 dex higher than those of the core collapse (CC) SNe hosts because the SNe Ia hosts contain a larger fraction of old stellar populations. Using the recent SN Ia delay-time distribution and the SFHs of the galaxies, we show that the SN Ia hosts in our sample are expected to produce twice as many SNe Ia as the CC SN hosts. Since both types occur in hosts with a similar SF rate and hence similar CC SN rate, this can explain the mass difference between the SN Ia and CC SN hosts, and reinforces the finding that at least part of the SNe Ia originate from very old progenitors. By comparing the mean SFH of the eight least massive galaxies with that of the massive SF SN Ia hosts, we find that the low-mass galaxies formed their stars during a longer time $(0.65 \%, 24.46 \%$, and $74.89 \%$ in the intervals $0-0.42 \mathrm{Gyr}, 0.42-2.4 \mathrm{Gyr}$, and $>2.4 \mathrm{Gyr}$, respectively) than the massive SN Ia hosts $(0.04 \%, 2.01 \%$, and $97.95 \%$ in these intervals). We estimate that the low-mass galaxies produce ten times fewer SNe Ia and three times fewer CC SNe than the high-mass group. Therefore the ratio between the number of CC SNe and SNe Ia is expected to increase with decreasing galaxy mass. CC SNe tend to explode at positions with younger stellar populations than the galaxy average, but the galaxy properties at $\mathrm{SNe}$ Ia locations are one average the same as the global galaxy properties.
\end{abstract}

Key words. supernovae: general - Galaxy: general - techniques: spectroscopic

\section{Introduction}

Supernova $(\mathrm{SN})$ explosions are one of the key processes that drive the chemical evolution of galaxies. Throughout their lifetime, stars fuse lighter into heavier chemical elements in their cores, and the explosion at the end of a star's life is responsible for dispersing the newly synthesized heavy elements into the interstellar medium (ISM). The next generation of stars form from gas that has already been enriched by heavier elements. Thus, starting from gas consisting of only $\mathrm{H}, \mathrm{He}$, and a tiny fraction of $\mathrm{Li}$, the heavy-element content of galaxies gradually increases to the present-day value of 2\% (Pagel 1997; Matteucci 2012).

Despite their key importance, the exact physical mechanisms that generate the explosions and the nature of the progenitor stars of SNe are not fully understood. It is generally accepted that in the final stages of their evolution, stars with initial masses heavier than $\sim 8 M_{\odot}$ lose their outer envelopes explosively. The explosion is triggered by the gravitational collapse of their heavy iron core into a neutron star or a black hole

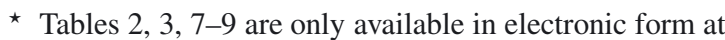
http: //www . aanda.org

$\star \star$ Corresponding author: L. Galbany,

e-mail: lluisgalbany@gmail.com
(Bethe et al. 1979; Arnett et al. 1989); these are collectively referred to as core-collapse $\mathrm{SNe}(\mathrm{CC} \mathrm{SNe})$. The end product of stars with masses between $\sim 0.5$ and $8 M_{\odot}$ is a degenerate carbon-oxygen (C/O) white dwarf (WD; Becker \& Iben 1980). The upper mass limit of C/O WDs is $\sim 1.1 M_{\odot}$ (Dominguez et al. 1999), but if such a star can increase its mass to $\sim 1.4 M_{\odot}$, thermonuclear reactions can ignite in the center and the WD can be completely disrupted in a very bright thermonuclear explosion that leads to a type Ia SN (SNe Ia, Hoyle \& Fowler 1960). $\mathrm{CC} \mathrm{SNe} \mathrm{disperse} \mathrm{large} \mathrm{amounts} \mathrm{of} \mathrm{intermediate} \mathrm{mass} \mathrm{elements}$ (IME) such as oxygen or carbon, but most of the synthesized iron-group elements remain locked into the compact degenerate remnants. On the other hand, a SN Ia produces few IME, but is a rich producer $\left(\sim 0.1-1 M_{\odot}\right)$ of iron and iron-peak elements (Mazzali et al. 2007).

In the past few decades, $\mathrm{SNe}$ Ia have become recognized as important cosmological probes. They are the best cosmological standard candles known to date. The observations of SNe Ia led to the discovery of the accelerating expansion of the Universe and dark energy (Riess et al. 1998; Perlmutter et al. 1999). This was possible because the empirically established tight relation of the light-curve shape to peak luminosity allowed measuring the luminosity distance with an accuracy of $\sim 7 \%$ (e.g., Phillips et al. 1999). 
In the future, SNe Ia will continue to play an essential role in the quest of unraveling the nature of dark energy (e.g., Albrecht et al. 2006). However, the $\mathrm{SNe}$ Ia technique is affected by systematic uncertainties, which need to be controlled to about $1 \%$ to distinguish between the different dark energy models. One such uncertainty stems from the fact that there has been little observational evidence of the exact evolutionary scenario that leads to the explosion. Theoretically, two channels through which SNe Ia can be created are suggested: the single-degenerate scenario (Whelan \& Iben 1973), where the WD accretes mass from a nondegenerate star, and the double-degenerate scenario, where two C/O WDs in a binary merge (Iben \& Tutukov 1984). However, no progenitor of a SN Ia has been unambiguously identified or observed (see Maoz et al. 2014, for a review). In both scenarios the total binary mass is expected to remain below $\sim 16 M_{\odot}$, and the lower limit is set by the requirement that the system is massive enough to allow the WD mass to reach $\sim 1.4 M_{\odot}$.

There have been several indications that the unknownprogenitor scenario might be an important source of uncertainty for cosmology. The deviation of the distance modulus inferred through the SNe Ia brightness from the expected value for a given cosmological model - the so-called Hubble residual correlates with some host galaxy parameters, such as the host galaxy mass (Sullivan et al. 2010; Lampeitl et al. 2010), the age of the host (Hamuy et al. 2000; Gupta et al. 2011), or the global metallicty (Gallagher et al. 2005). These galaxy parameters might in principle affect the properties of the WDs (i.e., the central density, metallicity, and C/O ratio, see Domínguez et al. 2001), which according to the numerical simulations of thermonuclear SN Ia explosions might in turn influence the peak luminosity, the light-curve width to luminosity relation and the colors of the resulting SNe (e.g., Höflich et al. 1998; Umeda et al. 1999; Domínguez et al. 2001; Röpke et al. 2006; Kasen et al. 2009; Bravo et al. 2010).

The $\mathrm{CC} \mathrm{SNe}$ are divided into three main subtypes depending on their spectral features around maximum-light. Type II (IIP/L/n) show $\mathrm{H}$ lines in their maximum light spectra, type $\mathrm{Ib}$ only $\mathrm{He}$, and type Ic lack both $\mathrm{H}$ and He lines ${ }^{1}$. This sequence reflects the state of the outer layers of the progenitor star at the moment of explosion. Type II SNe progenitors have kept their H-rich outer envelope intact, type Ib have lost the $\mathrm{H}$ envelope, and type Ic SN progenitors have lost both $\mathrm{H}-$ and He-rich layers. Indeed, the progenitors of some SNe IIP have been detected in pre-explosion images of nearby galaxies and indicate that they are red supergiants with masses 8.5-16.5 $M_{\odot}$ (for a review see Smartt 2009). However, no convincing detections of SN Ib/c progenitors are available (but see Gal-Yam et al. 2014; Cao et al. 2013; Bersten et al. 2014). There are two possible channels through which these explosions can occur. The first is through single, massive Wolf-Rayet stars (Gaskell et al. 1986; Crowther 2007) that have been stripped of their envelopes by strong radiation-driven winds. The other possibility is lowermass stars that lose their outer envelopes during evolution in a binary system (Podsiadlowski et al. 1992; Nomoto et al. 1996; Bersten et al. 2012).

Given the difficulties for direct detection, one of the available indirect methods to study the SN progenitors is through the properties of the host galaxies. Many such studies have been

\footnotetext{
Type IIb SNe are an intermediate class with $\mathrm{H}$ lines observed only in the early phases and He lines exclusively in the late phases. These $\mathrm{SNe}$ have only a very thin H-rich layer on the surface. We regard SNe IIb as part of the $\mathrm{SN} \mathrm{Ib/c} \mathrm{group.}$
}

performed, for example Hamuy et al. (1996, 2000), Gallagher et al. (2005, 2008), Sullivan et al. (2006, 2010), Prieto et al. (2008), Anderson \& James (2008), Raskin et al. (2009), Howell et al. (2009), Neill et al. (2009), Boissier \& Prantzos (2009), Kelly et al. (2010), Brandt et al. (2010), Anderson et al. (2010, 2012), Maoz et al. (2010, 2011), Leloudas et al. (2011), Modjaz et al. (2011), Kelly \& Kirshner (2012), Galbany et al. (2012), Kuncarayakti et al. (2013a,b), Rigault et al. (2013), Habergham et al. (2014), Shao et al. (2014), Hakobyan et al. (2014), which gave important insights about the properties of the different $\mathrm{SN}$ types and their progenitors. Most of these studies are based on analyses of the integrated or central host galaxies spectra, single-aperture or long-slit spectrographs to obtain spectra of the $\mathrm{SN}$ explosion sites, broad-band or narrow-band $\mathrm{H} \alpha$ imagery, SN rates or small field-of-view IFS that cover a small portion of the galaxy. Stanishev et al. (2012, hereafter S12) were the first who used large field-of-view (FoV) integral field spectroscopy (IFS) that covered the whole galaxy to study six SN Ia hosts at redshift $z \sim 0.02$. In this series of papers, we use observations of a larger sample of nearby SN host galaxies $(\langle z\rangle \sim 0.02)$ obtained with the same instrument by the CALIFA survey and several other programs (see Sect. 2).

With our wide-field IFS, we can derive spatially resolved two-dimensional (2D) maps of the host galaxy properties, which allows us not only to measure the properties at the SN position, but also to study how they are related to the overall distribution across the galaxy disk. Thus the main goals of our new study are to use IFS of nearby SN host galaxies to (i) improve SNe Ia as standard candles; and (ii) search for differences in environmental parameters to place more constraints on the nature of the progenitors of the different $\mathrm{SN}$ types. For this we study the relation of SN types to the properties of their local host galaxy environment at the SN position and the relation of the local to the global host properties and their distribution across the galaxies.

This paper, which is the first of the series, focuses on the galaxy properties that are related to the star formation and the galaxy star formation histories. The selection of the SN host galaxy sample used in this work is presented in Sect. 2. The methods used to extract the information needed for this study is outlined in Sect. 3. Our results are presented in Sect. 4, and the discussion of the results and our conclusions are finally presented in Sects. 5 and 6. Throughout the paper we assume the concordance cosmological model with $\Omega_{\mathrm{M}}=0.27, \Omega_{\Lambda}=0.73$, $w=-1$, and $h=0.708$.

\section{Galaxy sample and data reduction}

Here we use IFS of galaxies that hosted SNe where information on SN type is available. The observations come mainly from the CALIFA survey, although we also include data from several different sources, which are described below in more detail.

\subsection{CALIFA survey}

The CALIFA Survey (Sánchez et al. 2012a; Walcher et al. 2014) is an ongoing project that aims to obtain spatially resolved spectroscopic information of $\sim 600$ galaxies in the Local Universe $(0.005<z<0.03)$. The selection of targets was drawn from the 7th Data Release of the Sloan Digital Sky Survey (SDSS DR7, Abazajian et al. 2009) imposing the following restrictions:

(i) the targets need to be in the redshift range $0.005<z<0.03$; 
(ii) they need to have an angular isophotal diameter in the range $45<D_{25}<80$ arcsec to maximize the use of the large FoV of the integral field instrument; and

(iii) they are required to be at $\delta>7^{\circ}$ for galaxies in the North Galactic hemisphere to ensure good visibility from the observatory.

From the whole SDSS DR7 catalog, 939 galaxies passed these selection criteria and were included in what was called the CALIFA mother sample. This sample comprises galaxies of all morphological types (although spirals with and without bars dominate) and are well distributed across the entire colormagnitude diagram. More details on the survey, sample selection, and observational strategy are presented in Sánchez et al. (2012a) and Walcher et al. (2014). The first data release (Husemann et al. 2013) comprising IFS for 100 galaxies has been delivered to the community, and by June 2014 more than 450 objects were observed.

\subsubsection{Observations}

The observations are performed with the Potsdam Multi Aperture Spectograph (PMAS Roth et al. 2005) in PPAK mode (Verheijen et al. 2004; Kelz et al. 2006). The instrument is equipped with a $4 \mathrm{~K} \times 4 \mathrm{~K}$ E2V\#231 CCD and is mounted on the $3.5 \mathrm{~m}$ telescope of the Centro Astronomico Hispano-Aleman (CAHA) at the Calar Alto Observatory. The PPAK consists of a fiber bundle of 382 fibers with 2.7" diameter, 331 of which (science fibers) are ordered in a single hexagonal bundle with a filling factor of the FoV of 55\%. The remaining fibers are used for sky measurements (36), evenly distributed along a circle beyond the science fibers, and for calibration purposes (15).

Two overlapping setups are employed: the 500 lines $\mathrm{mm}^{-1}$ grating V500 with a spectral resolution of $\sim 6 \AA$ in the red (3750-7300 $\AA$ ) and the 1200 lines $\mathrm{mm}^{-1}$ grating V1200 in the blue (3400-4750 $\AA$ ) with a higher spectral resolution of $\sim 2.7 \AA$. The first setup provides a wider wavelength range, which allows studying properties of the stellar populations and the ionized gas, while the latter is intended for accurate measurements of both the stellar and ionized gas kinematics.

For each object, three $900 \mathrm{~s}$ exposures are obtained ${ }^{2}$. The second and third exposures are taken with an offset of $\Delta(\mathrm{RA}, \mathrm{Dec})=(-5.22,-4.84)$ and $(-5.22,+4.84)$ arcsec with respect to the first exposure to ensure that every point within the FoV is spectroscopically sampled. Combining these three pointings provides wavelength- and flux-calibrated $3 \mathrm{D}$ datacubes with $100 \%$ covering factor within a hexagonal FoV of $\sim 1.3 \operatorname{arcmin}^{2}$ with $1^{\prime \prime} \times 1$ " pixels, which correspond to $\sim 4000$ spectra per object. The data used in this work were reduced with version 1.4 of the CALIFA pipeline (García-Benito et al., in prep.).

On a given night, the observations are performed with only one of the grating configurations. A few galaxies were only observed with a single grating, but most were observed with both grating settings. When a target has been observed using both setups, the two 3D spectral datacubes are combined into a single datacube with the resolution of the V500 setup and covering the wavelength range $\sim 3650-7300 \AA$. The resulting combined cubes have a similar wavelength coverage as the V500 cubes, but with a higher signal-to-noise ratio $(\mathrm{S} / \mathrm{N})$ in the bluer part of the spectra. This is important for an accurate measurement of the [O II] $\lambda 3727$ emission line.

\footnotetext{
2 For V1200, each exposure is observed twice and then combined.
}

Table 1. SN selection from CALIFA observed galaxies.

\begin{tabular}{lccccc}
\hline \hline & II & Ibc/IIb & \multicolumn{2}{c}{ Ia } & \multirow{2}{*}{ All } \\
& & & SF & P & \\
\hline SNe in CALIFA hosts & 25 & 16 & 24 & 10 & 76 \\
Inside PPAK FoV & 22 & 13 & 17 & 6 & 58 \\
\hline
\end{tabular}

\subsubsection{SN host galaxies in CALIFA}

The coordinates of the whole CALIFA galaxy sample were compared with the International Astronomical Union (IAU) SN list ${ }^{3}$. We first selected galaxies that hosted a SN within 50 arcsec from the galaxy core ( FoV of PPAK) to have the spectrum of the host galaxy at the position of the SN. There were some cases where the SN exploded far from the center and outside the hexagonal shape of the FoV, and hence we excluded those targets (see Table 1). We performed a thorough search in the literature to reduce the effects of $\mathrm{SN}$ classification errors from the initial discoveries because classifications can often change after the initial discovery and therefore those in the catalog might not be completely accurate. The SN types and offsets from the galaxy nuclei were taken from the Asiago SN catalog ${ }^{4}$ (Barbon et al. 1989), except for two SNe (2005ip and 2006lc) marked in Table 2 for which the type was taken directly from the literature. We also visually inspected images of the $\mathrm{SNe}^{5}$ to verify the $\mathrm{SN}$ position.

Our CALIFA sample comprises 50 galaxies that hosted $58 \mathrm{SNe}$. Table 2 gives their details. The galaxies already publicly available in CALIFA DR1 are flagged with $\star$ in this table, while those that were only observed with the V500 grating are marked with $\dagger$.

\subsection{Other samples from PPAK/PMAS}

We expanded our CALIFA sample by adding other galaxies that were previously observed by different groups within the CALIFA collaboration. These observations were obtained with the same PMAS/PPAK instrument, but using different gratings: V300 and V600. The V300 grating covers the wavelength range of 3620-7056 $\AA$, providing a spectral resolution of $10.7 \AA$, and the V600 grating covers a wavelength range of 3845-7014 $\AA$ with a spectral resolution of $5.4 \AA$. Following the same procedure as for CALIFA galaxies, the galaxy coordinates were compared with the IAU SN list to find SNe within the FoV. The observations come from the following sources:

- The feasibility study for the CALIFA Survey (Mármol-Queraltó et al. 2011) obtained IFS of 48 nearby galaxies using V300 and V600 configurations. From this sample we selected four galaxies that hosted four $\mathrm{SNe}$ within the FoV.

- The PPAK IFS Nearby Galaxies Survey (PINGS Rosales-Ortega et al. 2010) observed 17 nearby disk galaxies using the V300 setup. Eight of these galaxies hosted eleven SNe.

- NGC 5668 (Marino et al. 2012) and NGC 3982 (Marino et al., in prep.), which were observed with the V300, hosted two and one SNe.

\footnotetext{
3 http://www. cbat.eps.harvard.edu/lists/Supernovae. html

4 Padova-Asiago Supernova Group web-page: http://graspa. oapd.inaf.it/

5 In http://www. rochesterastronomy.org/snimages/ and in the literature.
} 


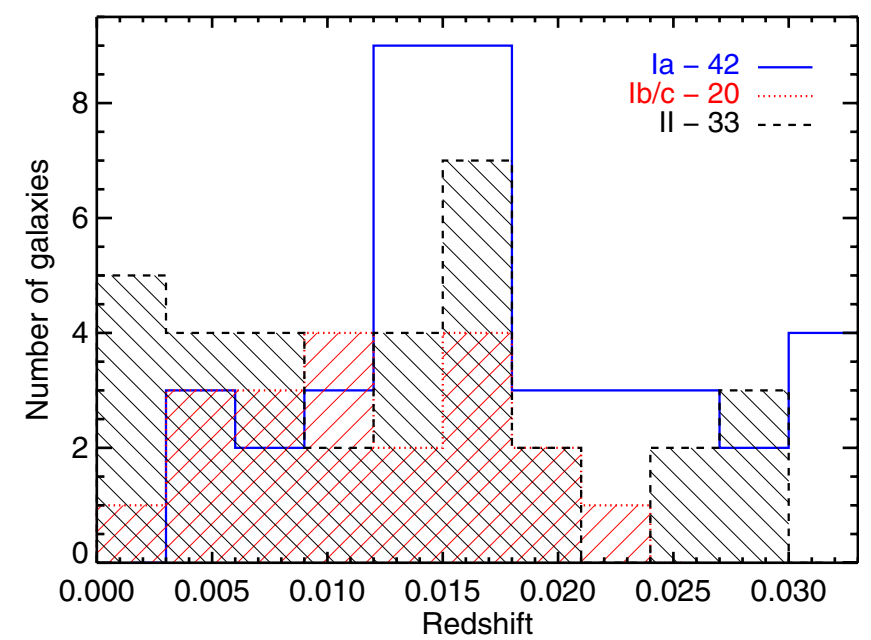

Fig. 1. Redshift distribution of the galaxies, colored according to the SN type they host.

- Five galaxies published in S12, observed with the V600 setup, which hosted six SNe Ia.

- Twelve galaxies that have been observed in several CALIFAextensions, proposals sent by members of the CALIFA collaboration (e.g., observations of interacting pair-galaxies; PIs: Barrera-Ballesteros, van de Ven, and García-Benito), and reduced in the same way as CALIFA data. They hosted $13 \mathrm{SNe}$.

The datacubes of all galaxies in S12, and NGC 0628, NGC 3184, and NGC 7319 from PINGS have a spatial resolution of $2^{\prime \prime}$ pixel $^{-1}$. The only difference when analyzing these cubes is that each pixel covers a larger physical area than the other galaxies in the sample at similar redshifts.

The SN types were obtained from the Asiago SN catalog except for SN 2002au, which was changed from Ia to IIb by Li et al. (2011) after fitting its light-curve, and for SN 1997ef and SN 2002ji which have been retyped by Modjaz et al. (2014) using optical spectra. In addition, SN $1961 \mathrm{~V}$ has been considered by some other works as a SN impostor. We kept it here following the conclusions of Smith et al. (2011) and Kochanek et al. (2012).

\subsection{Final sample}

We have split our SN sample into three groups: SNe Ibc/IIb that are the result of stripped-envelope progenitors, type II SNe whose progenitors have retained the outer hydrogen envelopes prior to explosion, and SNe Ia.

The total sample used in this work consists of $95 \mathrm{SNe}$ (33 SN II, $20 \mathrm{SN}$ Ibc/IIb, $42 \mathrm{SN}$ Ia) hosted by 81 galaxies. The galaxy and SN details for the whole sample are given in Tables 2 and 3. In Fig. 1 the redshift distribution of our sample for each SN type is shown, and in Table 4 their averages and standard deviations. Both CC SN redshift distributions have lower Kolmogorov-Smirnov (KS) test values than the SN Ia distribution. Targeted samples have shown low efficiency at detecting $\mathrm{CC} \mathrm{SNe}$ at redshifts higher than 0.02 , which explains the difference in the mean redshift of our subsamples. The upper panel of Fig. 2 shows the absolute $r$ magnitude $M_{r}$ versus redshift for the whole CALIFA mother sample and the galaxies used in this work, the lower panel shows $g-r$ color versus $M_{r}$. We used the SDSS magnitudes when available. Otherwise, $B$ and $V$ magnitudes from the literature obtained through the NED, SIMBAD, and Hyperleda databases were transformed into $g$
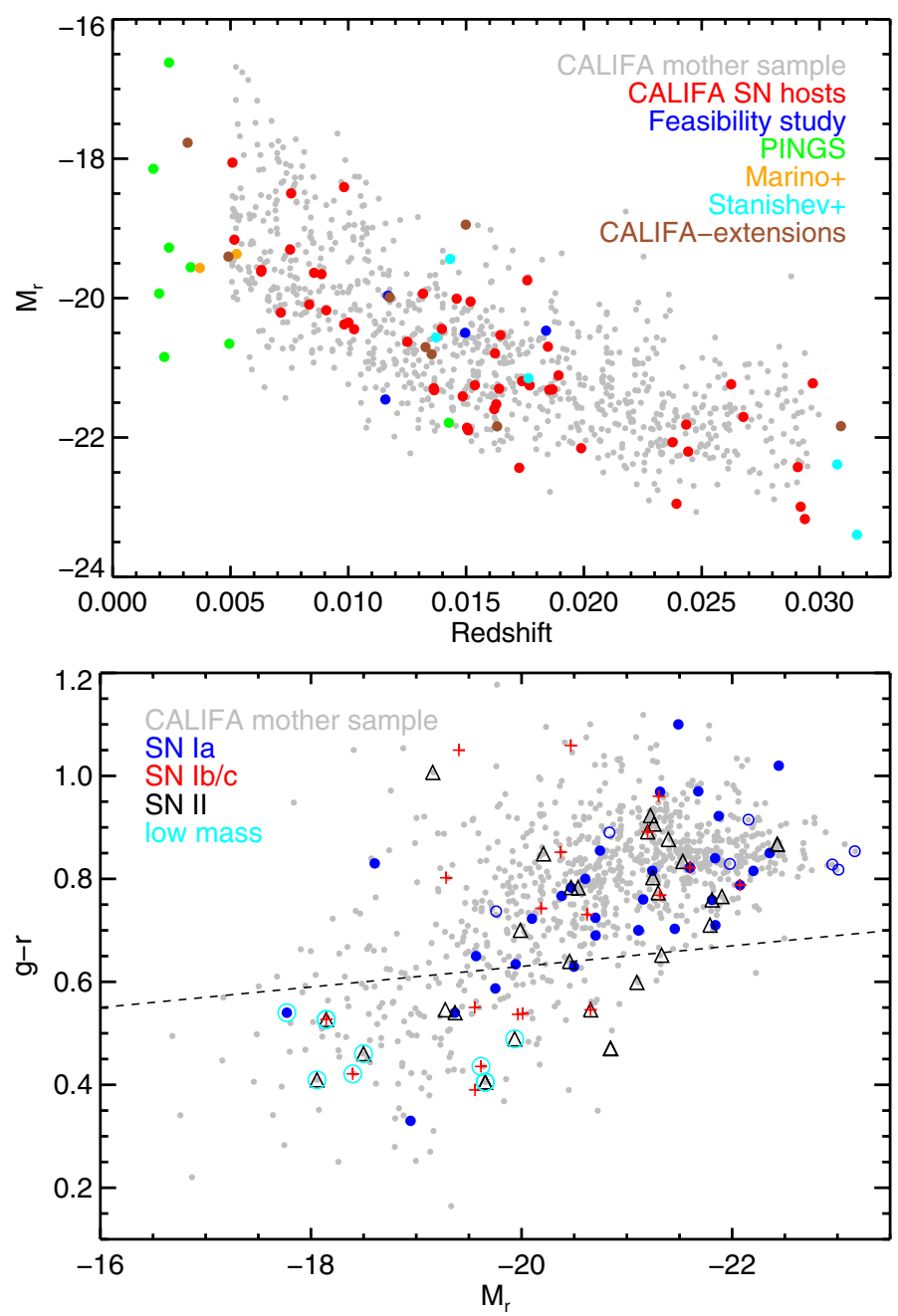

Fig. 2. Upper panel: absolute $r$-band magnitude $M_{r}$ vs. redshift of the whole CALIFA mother sample (gray dots) compared with the SN host galaxy sample used in this work from different sources (larger dots in different colors). Note that PINGS galaxies are more nearby than CALIFA galaxies. Lower panel: $g-r$ color vs. $M_{r}$ diagram of the CALIFA mother sample and the galaxies studied in this work. The dotted line shows the separation between blue from red galaxies proposed by Masters et al. (2010). The open blue circles show the passive SN Ia hosts (see Sect. 4). The points encircled with cyan open circles are the eight least massive galaxies in our sample (see Sect. 5.2), which are all blue.

and $r$ with the relations of Jester et al. (2005). NGC 6786, UGC 11975, and UGC 03555 are the only galaxies in our sample without magnitude information. In general, our sample follows the $M_{r}-z$ distribution of the CALIFA mother sample, with the exception of the PINGS galaxies, which are at lower redshift. According to the criterion of Masters et al. (2010) for separating red and blue galaxies, the CALIFA mother sample and our sample consist of $\sim 82 \%( \pm 1 \%)$ and $\sim 66 \%( \pm 5 \%)$ red galaxies. When our sample is separated into SN types, the hosts of SNe Ia are $\sim 83 \%( \pm 6 \%)$ red, while the hosts of SN Ib/c and II are about equally split $( \pm 7 \%)$ between red and blue galaxies, all errors calculated assuming binomial proportions.

\section{Data analysis of the IFS datacubes}

In our analysis, we closely followed the methods described in S12. We used our own programs written in IDL (updating those in S12) to extract the information of the spectra from 
Table 4. Statistics of the redshift distributions.

\begin{tabular}{ccccc}
\hline \hline & $\mathrm{Ia}$ & $\mathrm{Ib} / \mathrm{c}$ & $\mathrm{II}$ & $\mathrm{All}$ \\
\hline$\langle z\rangle$ & 0.0185 & 0.0116 & 0.0131 & 0.0152 \\
$\sigma_{z}$ & 0.0095 & 0.0060 & 0.0008 & 0.0089 \\
\hline
\end{tabular}

\begin{tabular}{lcccc}
\hline \hline & II - Ibc/Ilb & II - Ia & Ibc/IIb - Ia & CC - Ia \\
\hline K-S test & 0.915 & 0.116 & 0.024 & 0.013 \\
$(z<0.02)$ & 0.989 & 0.141 & 0.084 & 0.053 \\
\hline
\end{tabular}

the $3 \mathrm{D}$ datacubes and to derive $2 \mathrm{D}$ maps of the necessary parameters. This included the properties of the ionized gas and stellar populations. Before any automatic parameter extraction, we determined the galaxy center and the SN position within the 3D datacube. We summed the flux of both the columns and rows in the central box of 10 pixel side, and fitted a Gaussian to each of the profiles. The position in pixels of the peak was taken as the galaxy nucleus, and the SN position within the FoV was determined with the offset previously obtained with respect to the determined galaxy center. We applied spatial masks to CALIFA cubes by removing any region containing a spurious signal introduced by foreground stars and artifacts, that might affect both the stellar population fitting and the subsequent analysis of the galaxy properties. These masks were obtained from the SDSS $r$-band images using SExtractor (Bertin \& Arnouts 1996). All the original spectra were corrected for the Milky Way dust extinction ${ }^{6}$ using the dust maps of Schlegel et al. (1998) and applying the standard Galactic reddening law with $R_{V}=3.1$ (Cardelli et al. 1989; O'Donnell 1994). The spectra were then corrected to rest frame wavelengths.

The $\mathrm{S} / \mathrm{N}$ of each spectrum was determined in the wavelength window 4580-4640 $\AA$ as the inverse of $\sigma_{\text {diff }}$, the standard deviation of the difference between the signal and a third-order polynomial fit. Some spectra in the $3 \mathrm{D}$ datacubes had low $\mathrm{S} / \mathrm{N}$ for several reasons (vignetting, low $\mathrm{S} / \mathrm{N}$ in the outer galaxy regions). To increase the $\mathrm{S} / \mathrm{N}$ in these regions the pixels with low $\mathrm{S} / \mathrm{N}$ (but still with $S / N>1$ ) were combined into larger pixels with higher $\mathrm{S} / \mathrm{N}$. The binning was automatically derived using adaptive Voronoi tessellations (Cappellari \& Copin 2003; Diehl $\&$ Statler 2006) requiring the new combined pixels to have a $\mathrm{S} / \mathrm{N}$ of at least 20 in the continuum ${ }^{7}$ at $4610 \pm 30 \AA$. As a result, we lost spatial resolution in the outer galaxy regions, but the higher $\mathrm{S} / \mathrm{N}$ of the new combined spectra allowed us to measure the desired parameters when the $\mathrm{SN}$ was on a low $\mathrm{S} / \mathrm{N}$ pixel ${ }^{8}$. For 16 of all $95 \mathrm{SNe}$ the $\mathrm{S} / \mathrm{N}$ of the combined spectrum was not high enough to estimate the stellar population properties with confidence even after the Voronoi binning. For $70 \%$ of the remaining SNe little or no binning was needed and the automatic Voronoi binning worked well. For 23 of the SNe larger binning was required, and the automatic binning in general did not center the bin at the SN position. For the binned spectrum to better represent the galaxy properties at the SN position for these $23 \mathrm{SNe}$, a manual binning procedure was applied. The spectra were co-added within circular apertures centered on the SN position. For $15 \mathrm{SNe}$ the desired $\mathrm{S} / \mathrm{N}$ was reached for aperture radii up to three spaxels and only eight required radii as high

\footnotetext{
6 CALIFA datacubes are produced with the Galactic reddening correction applied.

7 It might be slightly lower because the covariance error form pixel to pixel. See Husemann et al. (2013) for more details.

8 The low $\mathrm{S} / \mathrm{N}$ is mostly a problem for stellar population analysis. For most galaxies the emission line fluxes at the $\mathrm{SN}$ position were measured without binning.
}

as six spaxels. The complete analysis, whose details are given in the following paragraphs, was made for each galaxy using both configurations, unbinned, Voronoi-binned, and mannually binned pixels, to check that our results are consistent. For each galaxy the total integrated spectrum was also computed by simply co-adding the spectra with $S / N>1$. This allowed us to compare the properties of the host as derived from integrated spectroscopy with those derived from spatially resolved spectroscopy. In total, 300 000 unbinned and 45000 co-added spectra were analyzed.

\subsection{Subtracting the stellar population spectrum}

In the spectrum of a galaxy, the emission lines are superimposed on the underlying stellar absorption spectrum. To accurately measure the emission line fluxes, the stellar continuum needs to be estimated and subtracted from the galaxy spectrum.

The star formation history of a galaxy can be approximated as the sum of discrete star formation bursts. Therefore, the observed stellar spectrum of a galaxy can be represented as the sum of spectra of single stellar populations (SSP) with different ages and possibly different metallicities. This in principle allows the stellar populations to be distinguished from the observed spectrum and therefore allows reconstructing the star formation history and chemical evolution of the galaxy.

We analyzed the stellar populations in the galaxies with STARLIGHT (Cid Fernandes et al. 2009, 2005; Mateus et al. 2006; Asari et al. 2007), a program that fits rest-frame galaxy spectra with a linear combination of model spectra of SSPs of different ages and metallicities. The contribution of the different SSPs that best describe the original spectrum can be used to study the properties of the galaxy stellar populations and estimate stellar velocity fields. This procedure has been adapted to the CALIFA data (Cid Fernandes et al. 2013, 2014), and the derivation of the galaxy mass and the spatially resolved star formation history and stellar mass surface density, ages, and stellar metallicities for the 100 galaxies in the CALIFA DR1 can be found in Pérez et al. (2013) and González Delgado et al. (2014b) for the CALIFA DR1 sample.

There have to be enough SSP models used as basis to reproduce the variability of different SSP for a given metallicity, but few enough so that the computing time can be minimized. We adopted a basis of 66 SSP components with 17 different ages (from 1 Myr to $18 \mathrm{Gyr}$ ) and four metallicities (0.2, 0.4, 1.0 and $2.5 Z_{\odot}$, where $Z_{\odot}=0.02$ ). They come from a slightly modified version of the models of Bruzual \& Charlot (2003) ${ }^{9}$, based on the MILES spectral library (Sánchez-Blázquez et al. 2006), Padova 1994 evolutionary tracks, Chabrier (2003) initial mass function (IMF) truncated at 0.1 and $100 M_{\odot}$, and new calculations of the TP-AGB evolutionary phase for stars of different mass and metallicity by Marigo \& Girardi (2007) and Marigo et al. (2008). The procedure of selecting the 66 SSPs from the whole set of 25 ages and six metallicities is detailed in S12.

Only spectra with $\mathrm{S} / \mathrm{N}$ greater than 5 at $4600 \AA$ were considered for STARLIGHT fits. The fit was restricted to a wavelength range from 3660 to $7100 \AA$ A. Some wavelength regions containing known optical nebular emission lines, telluric absorptions, or strong night-sky emission lines were masked out from the fit. The mean flux in the interval 4580-4620 ^ was used as a normalization factor for both the original spectrum and the model basis. Thus, STARLIGHT determines the fractional contribution of the different SSP models to the light at $\sim 4600 \AA, x_{i}$ and also

9 See Bruzual (2007) for more information. 


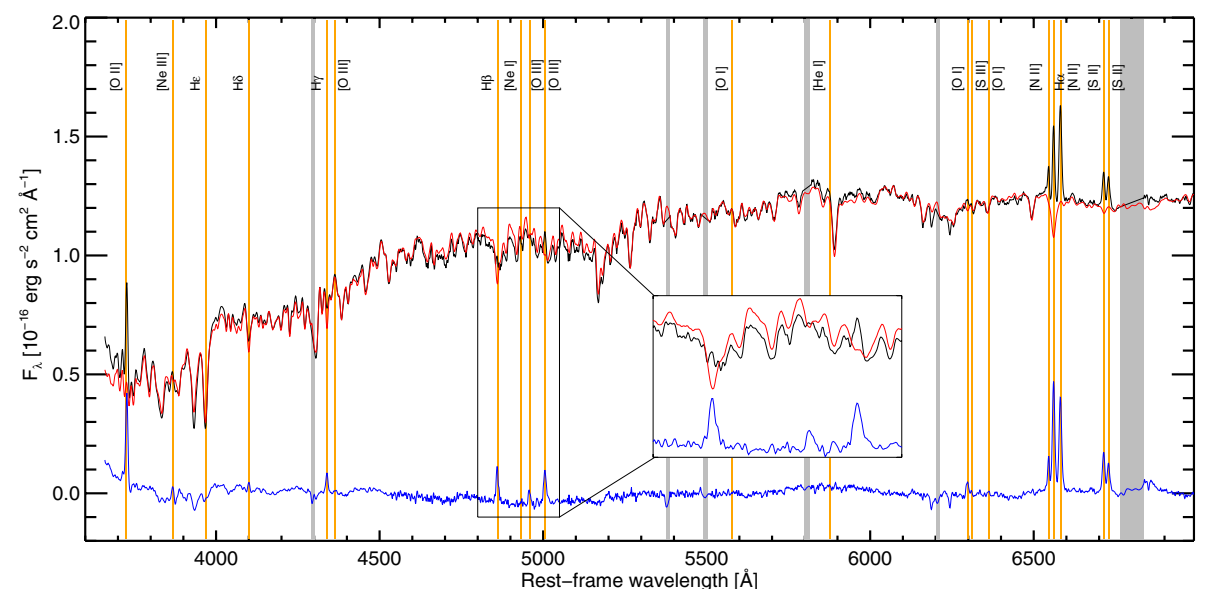

Fig. 3. Spectrum of NGC 2347 nucleus (black), together with the best STARLIGHT fit (red) and the pure nebular emission line spectrum (the difference, in blue). Note that the original spectrum is less noisy below $4500 \AA$, where the original V1200 spectrum has been degraded to the V500 resolution (6 ̊). Gray shadows are the wavelength ranges masked in the stellar population fit. Orange lines correspond the positions of the fitted emission lines. The region around $\mathrm{H} \beta$ and [O III] $\lambda 4959$ is zoomed in the inset to show that the emission lines are clearly seen only in the difference spectrum. Note also that the $[\mathrm{N} \mathrm{II}] \lambda 6583$ line in the observed spectrum is higher than $\mathrm{H} \alpha$, but it is lower in the continuum-subtracted spectrum. computes their contributions to the galaxy mass, $\mu_{i}$. In addition, the program also computes the total stellar mass, the velocity shift, and the Gaussian broadening that need to be applied to the model to fit the original spectrum. From the latter the velocity and dispersion maps for the stars can be produced. Different approaches are used to estimate the average age and metallicity of a spectrum (Sánchez-Blázquez et al. 2014; González Delgado et al. 2014a). Following Cid Fernandes et al. (2005), we can estimate the mean light weighted $(L)$ or mass weighted $(M)$ stellar age of the stellar population from

$$
\begin{aligned}
& \left\langle\log t_{*}\right\rangle_{L / M}=\sum_{i=1}^{N_{*}} w_{i} \log t_{i}, \\
& \left\langle Z_{*}\right\rangle_{L / M}=\sum_{i=1}^{N_{*}} w_{i} Z_{i},
\end{aligned}
$$

where $t_{i}$ and $Z_{i}$ are the age and the metallicity of the $i$ th SSP model, and $w_{i}=x_{i}$ or $w_{i}=\mu_{i}$ for light- and massweighted quantities, respectively. Compressed population vectors in three age bins were also computed: young $x_{\mathrm{Y}}$ (age < $300 \mathrm{Myr}$ ), intermediate $x_{\mathrm{I}}(300 \mathrm{Myr}<$ age $<2.4 \mathrm{Gyr}$ ), and old $x_{\mathrm{O}}$ (age $>2.4 \mathrm{Gyr}$ ).

In Fig. 3, the observed central spectrum of NGC 2347 and the STARLIGHT fit are shown. The fit subtraction gives the pure emission line spectrum. No $\mathrm{H} \beta$ emission line is seen in the observed spectrum (black), but after subtraction (blue), the line stands out. It can also be seen that the [N II] $\lambda 6583$ line in the observed spectrum is higher than the $\mathrm{H} \alpha$ emission, but it is lower in the continuum-subtracted spectrum. This example clearly shows the usefulness of subtracting the underlying stellar component before measuring the ionized gas emission lines (see also Kehrig et al. 2012).

\subsection{Ionized gas measurements}

\subsubsection{Emission line fluxes and extinction}

The STARLIHGT fits were subtracted from the observed spectra to obtain 3D cubes with the pure nebular emission line spectra. The most prominent emission lines were fitted using a weighted nonlinear least-squares fit with a single Gaussian plus a linear term. The area of the Gaussian was taken as an estimate of the line flux:

$F=\sqrt{2 \pi} \sigma I_{0}$,

where $\sigma$ and $I_{0}$ are the width and amplitude of the Gaussian. The uncertainty of the flux was determined from the $\mathrm{S} / \mathrm{N}$ of the measured line flux and the ratio between the fitted amplitude of the
Gaussian to the standard deviation of the adjacent continuum. Monte Carlo simulations were performed to obtain realistic errors to the line fluxes from these two measurements. For full details of the emission line flux measurement see Appendix C in S12.

The observed ratio of $\mathrm{H} \alpha$ and $\mathrm{H} \beta$ emission lines provides an estimate of the dust attenuation $A_{V}$ along the line of sight through a galaxy. Assuming an intrinsic ratio $I(\mathrm{H} \alpha) / I(\mathrm{H} \beta)=2.86$, valid for case B recombination with $T=$ $10000 \mathrm{~K}$ and electron density $10^{2} \mathrm{~cm}^{-3}$ (Osterbrock \& Ferland 2006), and using Fitzpatrick (1999) Milky Way extinction law, we obtained an estimate of $E(B-V)$. Adopting $R_{V}=A_{V} / E(B-$ $V)=3.1$ we calculated $A_{V}$. These calculations were made independently for each pixel to obtain 2D maps the extinction $A_{V}$. The emission lines measured previously were corrected for the dust extinction before calculating the line ratios and elemental abundances.

\subsubsection{Star formation rate}

We estimated the ongoing star formation rate (SFR) from the extinction-corrected $\mathrm{H} \alpha$ flux $F(\mathrm{H} \alpha)$ using the expression given by Kennicutt (1998b):

$\operatorname{SFR}\left[M_{\odot} \mathrm{yr}^{-1}\right]=7.9 \times 10^{-42} L(\mathrm{H} \alpha)$,

where

$L(\mathrm{H} \alpha)=4 \pi d_{L}^{2} F(\mathrm{H} \alpha)$

is the $\mathrm{H} \alpha$ luminosity in units of $\operatorname{erg~s}^{-1}, d_{\mathrm{L}}$ is the luminosity distance to the galaxy assuming a flat $\Lambda$ CDM cosmology with $\Omega_{\mathrm{M}}=0.27$ and $\Omega_{\Lambda}=0.73$, and $H_{0}=70.8 \mathrm{~km} \mathrm{~s}^{-1} \mathrm{Mpc}^{-1}$. This measurement was used to generate 2D maps of the specific SFR (sSFR) and the SFR density ( $\Sigma$ SFR), dividing in each pixel the calculated SFR over the stellar mass in that pixel and the pixel area in $\mathrm{kpc}^{2}$.

\subsubsection{AGN contribution}

Some methods used to derive relevant quantities can only be applied if the ionization source exclusively arises from the stellar radiation. To identify AGN contamination in the galaxy centers we used the so-called BPT diagnostic diagram (Baldwin et al. 1981; Veilleux \& Osterbrock 1987). The BPT diagram is a plot of $O 3 \equiv \log _{10}\left(\frac{[\mathrm{OIII}] \lambda 5007}{\mathrm{H} \beta}\right)$, and $N 2 \equiv \log _{10}\left(\frac{[\mathrm{N} \mathrm{II}] \lambda 6583}{\mathrm{H} \alpha}\right)$, on which gas ionized by different sources occupies different areas. Two criteria commonly used to separate star-forming (SF) 

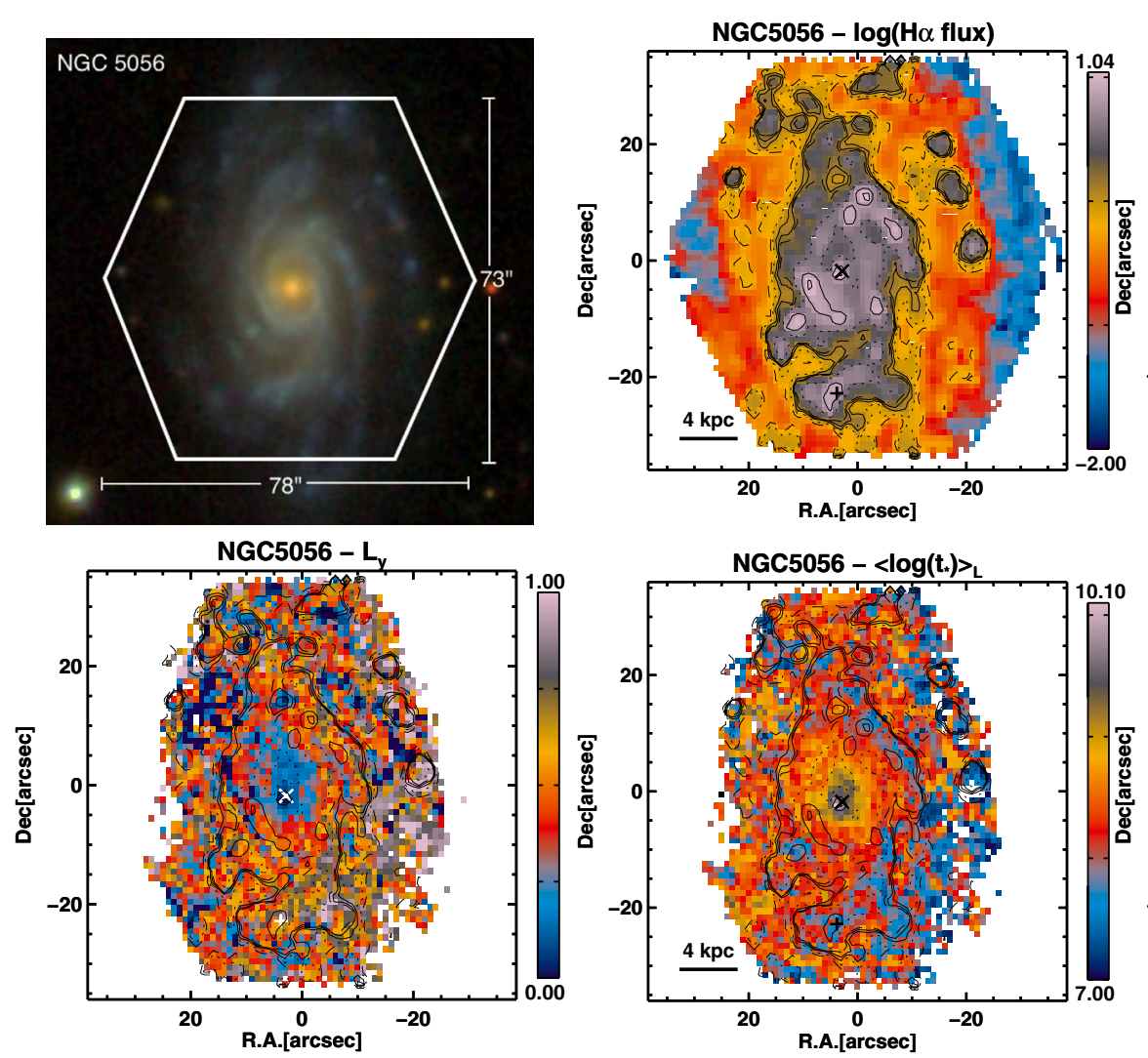

NGC5056 - H $\alpha$ velocity
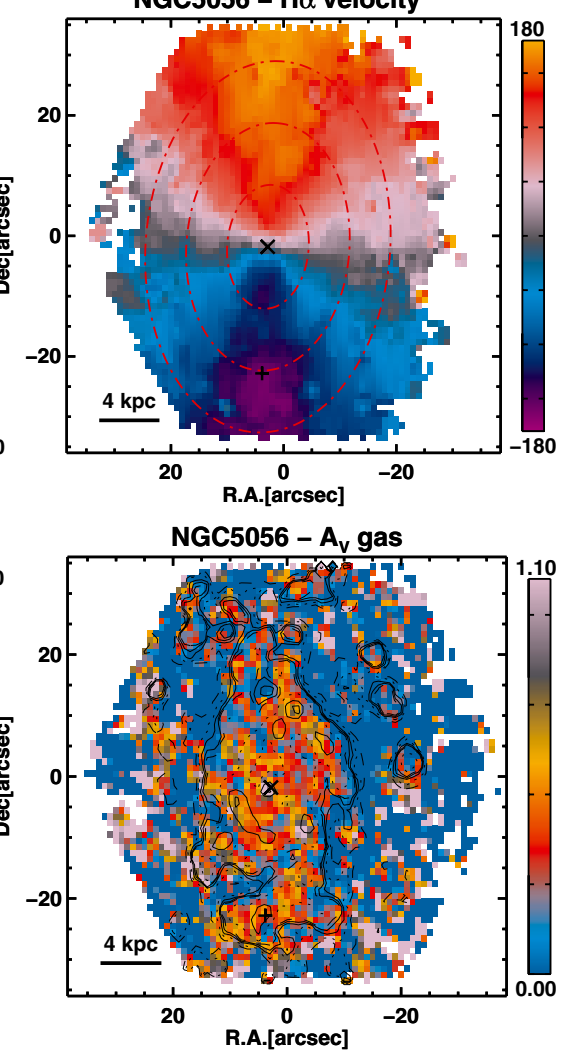

Fig. 4. Top: from left to right, SDSS image of NGC 5056 with the PPAK aperture superimposed on scale; two-dimensional maps of the extinctioncorrected $\mathrm{H} \alpha$ flux and the velocity measured from the $\mathrm{H} \alpha$-line shift. Bottom: from left to right, two-dimensional maps of the contribution of the young stars $(<100 \mathrm{Myr})$ to the total luminosity, the light-weighted average stellar age, and the extinction $\left(A_{V}\right)$. In all 2D maps with IFS data, $\times$ determines the galaxy center and + the SN position. The contour levels overplotted on the maps are derived from the H $\alpha$ flux map. The $x-y$ coordinates are in arcsec with respect to the center of the FoV. The orientation of the images is north up, east left.

from AGN-dominated galaxies are the expressions in Kewley et al. (2001) and (Kauffmann et al. 2003). However, it should be noted that the latter is an empirical expression, and bona fide $\mathrm{H}$ II regions can be found in the composite area determined by it (Sánchez et al. 2014). In addition, the AGN region can be separated into Seyfert and LINER regions using the expression in Cid Fernandes et al. (2010):

$O 3=1.01 \times N 2+0.48$.

Central pixels falling in the AGN-dominated region according to the criterion of Kewley et al. (2001) were excluded from the analysis.

\subsection{Distance deprojection and azimuthal average}

The $\mathrm{H} \alpha$ line shift from the expected position provides the best estimate of the gas velocity field. The fields were analyzed with the methods and IDL programs developed by Krajnović et al. (2006). The program analyzes the velocity field at several radii and for each of them returns the orientation angle (PA) and the axes ratio (b/a) and quantifies the degree of deviation from a pure disk rotation. Results for all galaxies are listed in Table 2. For galaxies without emission lines and for which the $\mathrm{H} \alpha$ emission could not be measured, PA and b/a were measured from the star velocity map previously obtained from the STARLIGHT fit. From the PA and b/a kinematic parameters the deprojected galactocentric distances (GCD) of each pixel in the field of view were also computed. The 2D maps of the deprojected GCD distances were used to study the radial dependencies of all measured parameters.

To verify the representativeness of our SN samples we compared their radial distributions with those derived from the Asiago SN catalog, using two different measurements of the galactocentric distance (GCD):

1. The projected (observed) GCD is a lower limit of the real distance. This is the best approximation to the real distance when no information on the inclination and the orientation of the galaxy is available. The projected GCD is measured using the offset of the $\mathrm{SN}$ from the galaxy center in arcsec and converted into kpc using its redshift. It has been previously used in several studies, for example, Ivanov et al. (2000) and Galbany et al. (2012).

2. The deprojected $G C D$, recovered from the inclination and the orientation of the galaxy, which is the best estimate for the real GCD if the SN lies in the galactic plane. Examples of previous works using this approach are Hakobyan et al. (2009), Wang et al. (2010), Galbany et al. (2012) and Kangas et al. (2013). The galaxy inclinations and orientations were obtained from an analysis of the $2 \mathrm{D} \mathrm{H} \alpha$ velocity maps using the procedure previously described in Sect. 3.3.

We selected only SNe for which the type, redshift, PA, b/a, and offset from the nucleus were available. Furthermore, to mimic our sample as closely as possible, we selected only $\mathrm{SNe}$ at $z<0.03$ and apparent GCD lower than 40". Figure 5 shows the cumulative distributions for the three SN subgroups in our 


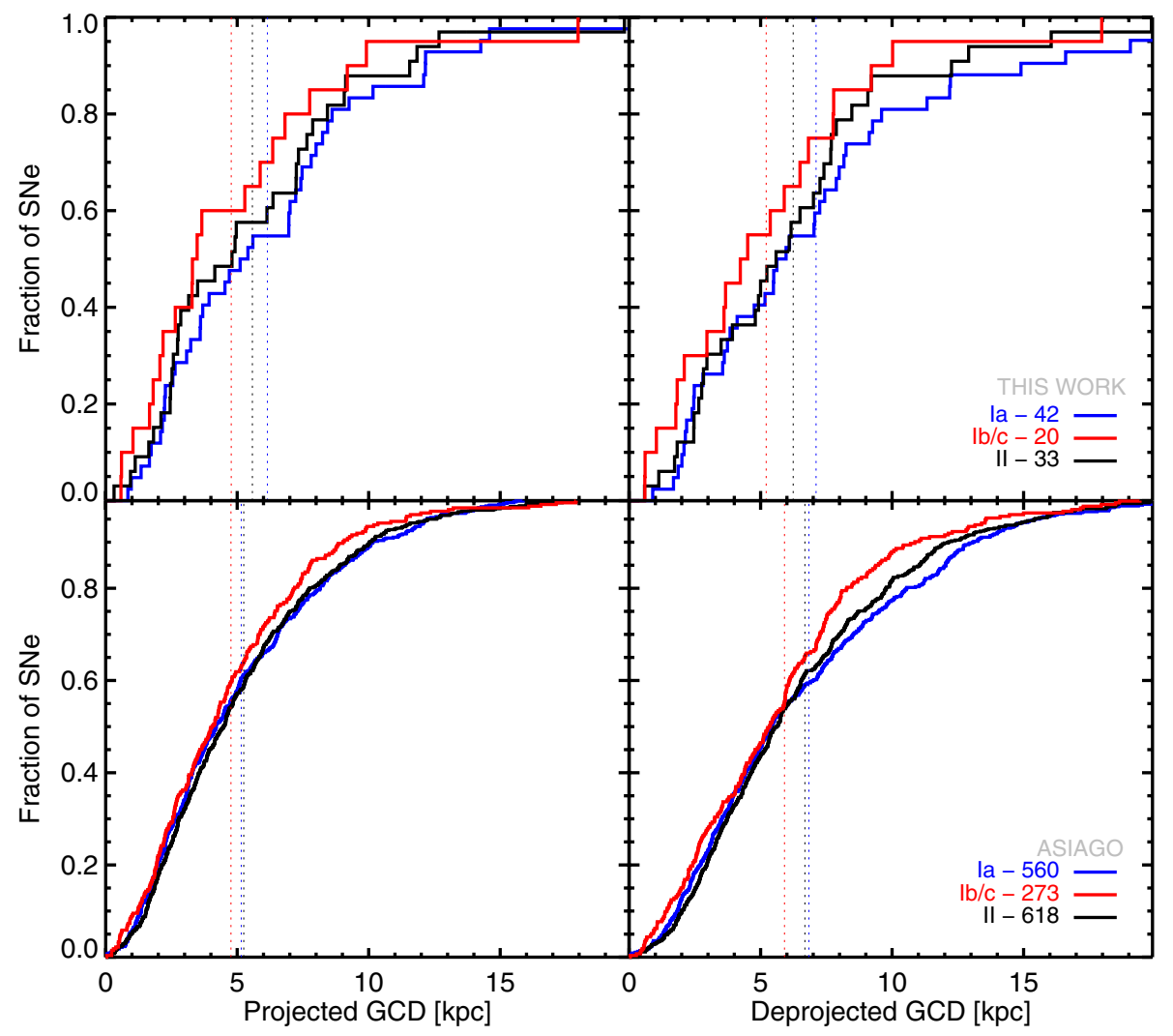

Fig. 5. Cumulative distributions of GCD measurements. Top: projected GCD in kpc (left), and deprojected GCD in kpc (right). Bottom: as a comparison, the same plots with $\mathrm{SNe}$ from the Asiago SN catalog. The ordinate indicates the fraction of the SN population with GCD lower than the abscissa value, and vertical lines show the mean value of each distribution. sample and from the Asiago catalog. The mean values of the distributions agree very well between our and Asiago catalog samples. Furthermore, we performed two-sample K-S tests between our and Asiago samples for each of the three SN subtypes to check that the two samples are drawn from a single underlying distribution. The obtained high $p$-values $(>0.1)$ imply that the two samples are drawn from similar populations, and we conclude that the radial distribution of our SN sample is not heavily biased. Finally, we repeated the exercise by restricting the sample to SNe only in spiral galaxies, and both the statistics and the $\mathrm{K}-\mathrm{S}$ tests gave similar results.

\section{Results}

Although narrow-band $\mathrm{H} \alpha$ imaging has been used in the past to study the association of different SN types with the SF (James \& Anderson 2006; Anderson \& James 2008), IFU spectroscopy has several critical advantages. The $\mathrm{H} \alpha$ line flux, which provides one of the most accurate estimates of the ongoing SFR (Kennicutt 1998a), can be measured much more accurately from spectra. Additionally, star formation is almost always associated with dust, and the light emerging from star-forming regions often shows signs of considerable dust reddening. By measuring the $\mathrm{H} \alpha$ and $\mathrm{H} \beta$ fluxes the amount of dust extinction can be estimated. This enables studying the association of different SN types with the absolute ongoing SFR measured from the $\mathrm{H} \alpha$ flux corrected for dust extinction. While the $\mathrm{H} \alpha$ flux provides an estimate of the ongoing SFR ( $<10 \mathrm{Myr})$, an analysis of the whole spectrum, for example, by the full spectrum-fitting technique, can provide much richer information on the SFH of the galaxy and the properties of its stellar populations.

In this section we explore the $2 \mathrm{D}$ maps of $\mathrm{SN}$ host galaxies in search for correlations between the SN type and the properties of their host galaxies regarding star formation and stellar populations. Seven of the galaxies in our sample, each of them host of one SN Ia, can be considered as passive based on their morphology and total SFR: NGC 0495, NGC 4874, NGC 5611, NGC 1060, NGC 6166, NGC 6173, and UGC 10097. After subtracting the stellar continuum contribution, the first three do not show emission lines at any position. The remaining four only show weak emission in their central regions (Papaderos et al. 2013; Singh et al. 2013). In NGC 1060, NGC 6166, and UGC 10097 this nuclear emission was strong enough to be detected in the total spectra as well. For these passive galaxies it was not possible to obtain the emission-line-based spectroscopic parameters at the $\mathrm{SN}$ positions. In the following subsections, we consider these seven passive galaxies only when analyzing the total galaxy properties and the results obtained from the analysis of the stellar populations at the SN position.

Below, we first compare the global properties of the host galaxies of the three SN subtypes and then analyze the galaxy properties at the SN positions. The measured quantities for the individual galaxies/SNe are shown in Tables 7-9. The means and medians of the distributions and their asymmetric errors are given in Table 5 for the three SN subtypes, the $p$-values of the KS tests are listed in Table 6.

\subsection{Global SN host galaxy properties}

From 3D data-cubes a galaxy total ongoing SFR can be measured in two ways: from $\mathrm{H} \alpha$ flux in the total spectrum and by summing the SFR estimates in all individual spaxels. These two estimates agree well. However, for a SFR lower than a few $M_{\odot} \mathrm{yr}^{-1}$ the estimates from summing the 2D maps are higher by up to $50 \%$ that the estimates from the total spectrum. This is most likely a result from the emission-line-fitting procedure, where a zero lower limit of the line amplitudes is imposed. In spaxels that contain zero or close to zero $\mathrm{H} \alpha$ flux one can 
L. Galbany et al.: Nearby supernova host galaxies from the CALIFA Survey. I.

Table 5. Mean and median values with asymmetric standard deviations of the distributions of the host galaxy properties for the three SN types: global properties derived from the total spectrum, and local properties at the SN positions.

\begin{tabular}{|c|c|c|c|c|}
\hline \multirow[t]{2}{*}{ SN type } & \multicolumn{2}{|c|}{ Global } & \multicolumn{2}{|c|}{ Local } \\
\hline & mean & median & mean & median \\
\hline \multicolumn{5}{|c|}{$\log \left(M_{*}\left[M_{\odot}\right]\right)$} \\
\hline Ia-all & $10.78(-0.49,+0.46)$ & $10.82(-0.51,+0.55)$ & - & - \\
\hline $\mathrm{Ia}-\mathrm{SF}$ & $10.67(-0.38,+0.45)$ & $10.67(-0.38,+0.45)$ & - & - \\
\hline $\mathrm{b} / \mathrm{c}$ & $10.24(-0.58,+0.58)$ & $10.28(-0.47,+0.54)$ & - & - \\
\hline II & $10.39(-1.05,+0.60)$ & $10.48(-0.80,+0.52)$ & - & - \\
\hline & \multicolumn{2}{|c|}{$\begin{array}{c}\log (\text { total } S F R) \\
{\left[M_{\odot} \mathrm{yr}^{-1}\right]}\end{array}$} & \multicolumn{2}{|c|}{$\begin{array}{c}\log \left(\Sigma S F R_{\mathrm{SN}}\right) \\
{\left[M_{\odot} \mathrm{yr}^{-1} \mathrm{kpc}^{-2}\right]}\end{array}$} \\
\hline$\overline{\text { Ia-all }}$ & $-0.16(-1.04,+0.67)$ & $0.17(-0.80,+0.43)$ & $-2.54(-0.61,+0.97)$ & $-2.77(-0.39,+1.12)$ \\
\hline $\mathrm{Ia}-\mathrm{SF}$ & $0.18(-0.46,+0.44)$ & $0.19(-0.47,+0.43)$ & $-2.45(-0.85,+0.96)$ & $-2.57(-0.73,+1.00)$ \\
\hline & $0.18(-0.53,+0.40)$ & $0.24(-0.39,+0.34)$ & $-1.80(-0.48,+0.44)$ & $-1.72(-0.56,+0.36)$ \\
\hline & $0.20(-0.29,+0.56)$ & $0.14(-0.40,+0.57)$ & $-2.06(-0.77,+0.57)$ & $-2.06(-0.76,+0.57)$ \\
\hline & \multicolumn{4}{|c|}{$\left\langle\log t_{*}\right\rangle_{L}[\mathrm{yr}]$} \\
\hline Ia-all & $9.24(-0.45,+0.78)$ & $9.20(-0.41,+0.82)$ & $9.12(-0.52,+0.73)$ & $9.07(-0.47,+0.79)$ \\
\hline $\mathrm{Ia}-\mathrm{SF}$ & $9.07(-0.28,+0.54)$ & $9.03(-0.24,+0.53)$ & $8.98(-0.42,+0.39)$ & $8.94(-0.38,+0.43)$ \\
\hline & $8.71(-0.56,+0.33)$ & $8.94(-0.67,+0.10)$ & $8.36(-1.29,+0.63)$ & $8.65(-0.96,+0.36)$ \\
\hline \multirow[t]{2}{*}{ II } & $8.80(-0.64,+0.47)$ & $8.82(-0.66,+0.45)$ & $8.55(-0.64,+0.71)$ & $8.51(-0.60,+0.63)$ \\
\hline & \multicolumn{4}{|c|}{$x_{\mathrm{Y}}[\%]$} \\
\hline Ia-all & $17.3(-14.1,+19.5)$ & $14.4(-12.2,+22.3)$ & $21.1(-17.9,+28.2)$ & $15.2(-12.3,+28.2)$ \\
\hline $\mathrm{Ia}-\mathrm{SF}$ & $20.5(-12.2,+18.9)$ & $15.0(-8.6,+21.8)$ & $24.1(-19.1,+27.8)$ & $18.1(-14.6,+25.3)$ \\
\hline $\mathrm{b} / \mathrm{c}$ & $26.8(-11.9,+11.7)$ & $24.6(-9.7,+8.9)$ & $41.0(-21.9,+30.7)$ & $29.1(-10.0,+42.6)$ \\
\hline \multirow[t]{2}{*}{ II } & $26.6(-12.0,+22.5)$ & $24.8(-13.8,+20.3)$ & $35.8(-16.5,+32.2)$ & $33.5(-16.6,+16.5)$ \\
\hline & \multicolumn{4}{|c|}{$x_{\mathrm{I}}[\%]$} \\
\hline Ia-all & $26.6(-26.6,+21.3)$ & $25.9(-25.9,+22.0)$ & $31.4(-25.6,+34.3)$ & $26.3(-22.8,+35.8)$ \\
\hline $\mathrm{Ia}-\mathrm{SF}$ & $31.3(-23.1,+24.2)$ & $32.4(-24.3,+23.1)$ & $35.9(-25.8,+29.8)$ & $31.0(-21.0,+31.1)$ \\
\hline $\mathrm{b} / \mathrm{c}$ & $38.8(-13.3,+15.3)$ & $42.0(-16.5,+12.1)$ & $29.1(-29.1,+24.9)$ & $27.4(-27.4,+26.6)$ \\
\hline \multirow[t]{2}{*}{ II } & $30.4(-17.8,+20.5)$ & $32.9(-20.3,+18.0)$ & $32.0(-32.0,+24.9)$ & $32.1(-30.2,+24.9)$ \\
\hline & \multicolumn{4}{|c|}{$x_{\mathrm{O}}[\%]$} \\
\hline Ia-all & $56.1(-24.8,+34.3)$ & $52.4(-21.1,+38.0)$ & $47.5(-33.0,+39.3)$ & $45.8(-31.3,+41.0)$ \\
\hline $\mathrm{Ia}-\mathrm{SF}$ & $48.3(-21.8,+25.1)$ & $46.7(-20.2,+26.6)$ & $40.1(-27.1,+30.4)$ & $36.2(-23.3,+31.5)$ \\
\hline $\mathrm{b} / \mathrm{c}$ & $34.4(-18.1,+15.9)$ & $35.0(-18.7,+15.3)$ & $29.9(-15.0,+33.2)$ & $26.2(-11.3,+19.8)$ \\
\hline \multirow[t]{2}{*}{ II } & $43.0(-22.0,+19.6)$ & $43.5(-22.6,+19.1)$ & $32.1(-24.0,+28.9)$ & $27.5(-19.4,+25.4)$ \\
\hline & \multicolumn{4}{|c|}{$\mathrm{H} \alpha E W[\AA]$} \\
\hline Ia-all & $11.8(-11.5,+11.0)$ & $11.9(-11.3,+10.9)$ & $12.7(-11.7,+17.4)$ & $7.8(-7.2,+18.1)$ \\
\hline $\mathrm{Ia}-\mathrm{SF}$ & $14.1(-9.3,+10.0)$ & $13.0(-8.9,+11.1)$ & $15.2(-11.5,+14.9)$ & $10.2(-6.9,+17.8)$ \\
\hline $\mathrm{b} / \mathrm{c}$ & $26.0(-10.4,+13.0)$ & $18.3(-4.4,+15.7)$ & $57.3(-41.8,+33.7)$ & $38.0(-22.6,+39.7)$ \\
\hline II & $22.8(-10.9,+32.0)$ & $20.2(-9.0,+14.8)$ & $36.7(-21.8,+40.3)$ & $31.9(-17.6,+25.7)$ \\
\hline
\end{tabular}

Table 6. P-values of the KS test for all measurements in Sect. 4.

\begin{tabular}{lccrrr}
\hline \hline & II-Ibc & II-Ia(SF) & II-Ia & Ibc-Ia(SF) & Ibc-Ia \\
\hline \multicolumn{5}{c}{ Global } \\
$\log$ Mass & 0.351 & 0.201 & 0.166 & 0.020 & 0.015 \\
$\log S F R$ & 0.915 & 0.825 & 0.594 & 0.999 & 0.707 \\
$\left\langle\log t_{*}\right\rangle_{L}$ & 0.404 & 0.108 & 0.043 & 0.068 & 0.005 \\
$x_{\mathrm{Y}}$ & 0.428 & 0.004 & 0.000 & 0.068 & 0.005 \\
$x_{\mathrm{I}}$ & 0.330 & 0.856 & 0.414 & 0.147 & 0.031 \\
$x_{\mathrm{O}}$ & 0.344 & 0.708 & 0.084 & 0.209 & 0.016 \\
$\mathrm{H} \alpha E W$ & 0.688 & 0.131 & 0.012 & 0.013 & 0.002 \\
\hline \multicolumn{5}{c}{ Local } & \\
$\log \Sigma S F R$ & 0.260 & 0.135 & - & 0.015 & - \\
$\left\langle\log t_{*}\right\rangle_{L}$ & 0.535 & 0.018 & 0.004 & 0.074 & 0.011 \\
$x_{\mathrm{Y}}$ & 0.584 & 0.018 & 0.004 & 0.082 & 0.021 \\
$x_{\mathrm{I}}$ & 0.915 & 0.919 & 0.836 & 0.592 & 0.963 \\
$x_{\mathrm{O}}$ & 0.698 & 0.713 & 0.298 & 0.164 & 0.038 \\
$\mathrm{H} \alpha E W$ & 0.428 & 0.003 & - & 0.001 & - \\
\hline
\end{tabular}

measure both positive and negative flux with equal probability because of the noise. The negative fluxes are set to zero, therefore the noisy spaxels will only contribute with positive flux to the total sum, wichh leads to an overestimation of the total $\mathrm{H} \alpha$ flux. This effect will be stronger for galaxies with a low SFR, which are most of the galaxies in our sample. For this reason we used the SFR estimate from the total spectrum.

Figure 6 shows the cumulative distribution of the total SFR. Two distributions are plotted for $\mathrm{SNe}$ Ia, one with all galaxies and one with only the late-type SF galaxies ${ }^{10}$. CC SNe explode only in SF galaxies. From Fig. 6 one can see that on average there is no difference between the total ongoing SFR in CC and $\mathrm{SNe}$ Ia hosts when SF galaxies alone are considered. For the three $\mathrm{SN}$ types the mean $\log (S F R) \simeq+0.2$ corresponding to $S F R \simeq 1.6 M_{\odot} \mathrm{yr}^{-1}$. We performed two-sample KolmogorovSmirnov (K-S) tests between the samples and found a high probability $(>0.8)$ for them to come from the same population. When the passive galaxies are included, the distribution for SNe Ia is of course shifted toward a lower SFR.

The total galaxy stellar mass was estimated from the STARLIGHT fits of the total spectra. The cumulative distributions in Fig. 7 show that the hosts of SNe Ia are more massive than those of CC SNe. When only SF galaxies are considered, the SN Ia hosts are more massive by $\sim 0.3$ dex than SN II hosts, and the difference increases to $\sim 0.4$ dex for the whole SN Ia sample.

\footnotetext{
${ }^{10}$ Some of the early-type galaxies do show traces of weak nuclear SFR, but nonetheless we consider them as passive here.
} 


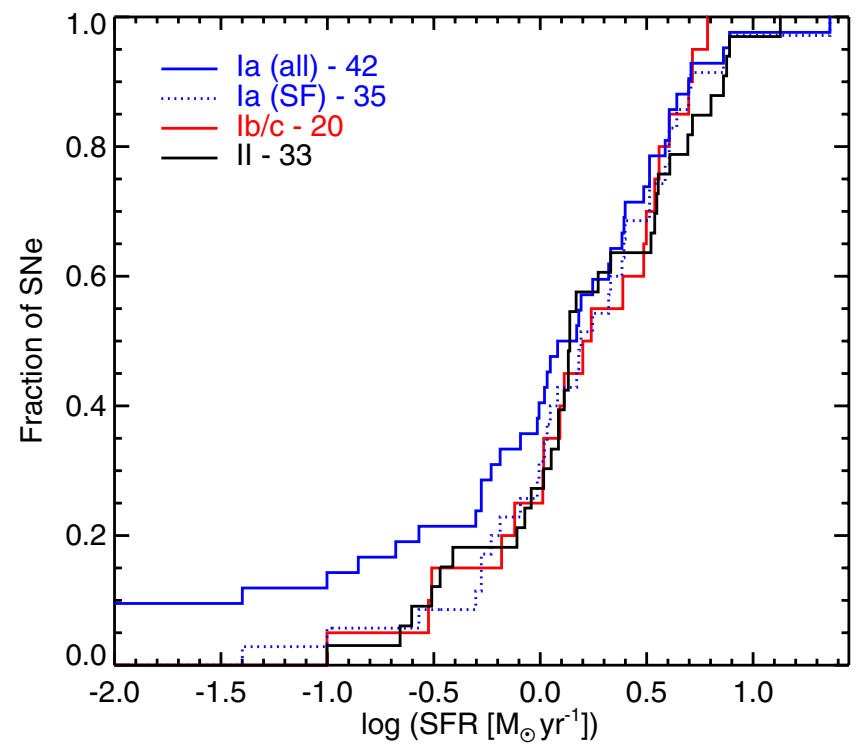

Fig. 6. Cumulative distribution of the ongoing total SFR in the host of the three main SN types. In this and the following CD plots the ordinate indicates the fraction of $\mathrm{SN}$ with values lower than the abscissa value. In some plots the vertical lines show the mean value of each distribution.

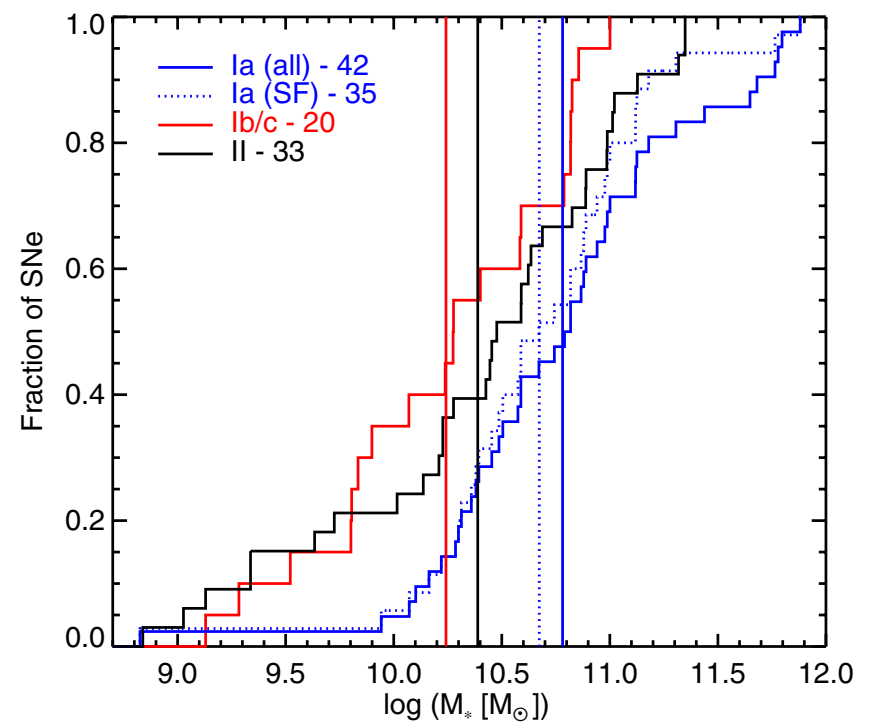

Fig. 7. Cumulative distributions of the galaxy stellar mass for the three $\mathrm{SN}$ types. Two lines are shown for $\mathrm{SNe} \mathrm{Ia}$, one for all galaxies, and another one for only the SF galaxies.

There is also evidence that the hosts of $\mathrm{SNe} \mathrm{Ib} / \mathrm{c}$ are $\sim 0.1 \mathrm{dex}$ lighter than those of SNe II.

It is interesting to note that in our SN Ia sample only one galaxy has a mass significantly lower than $10^{10} M_{\odot}$, while $\sim 30 \%$ of the CC SNe are in galaxies with such low masses. It is wellknown that lower-mass galaxies are under-represented in the nearby SN Ia sample (Kelly et al. 2010; Neill et al. 2009; Arcavi et al. 2010). This is usually attributed to the fact that nearby SNe Ia are discovered by search programs that target bright massive galaxies. However, this cannot explain why $\sim 30 \%$ of the CC SNe are found in galaxies with mass $M_{*} \leq 10^{10} M_{\odot}$. Nearby $\mathrm{CC} \mathrm{SNe}$ are found by the same search programs that find SNe Ia, and more SNe Ia should have been found in lower-mass galaxies.

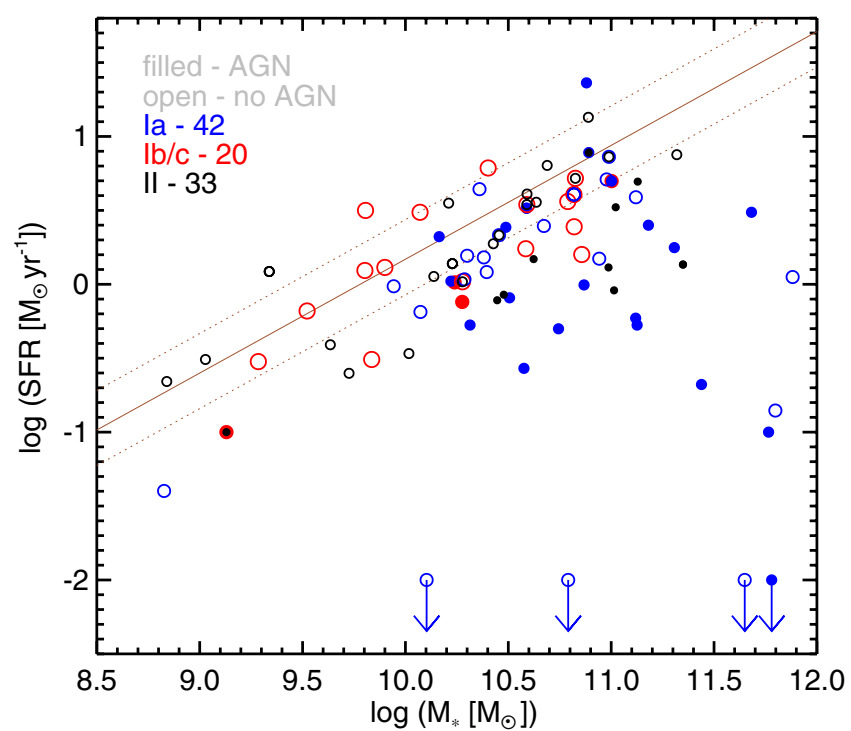

Fig. 8. Total SFR-mass relation. Dotted lines show the locus described in Elbaz et al. (2007) for galaxies at $z \sim 0$.

Figure 8 shows the ongoing SFR versus the stellar mass. Most of the SF galaxies lie along the locus determined by Elbaz et al. (2007) using SDSS data at $z \sim 0$ (see also Brinchmann et al. 2004). It is interesting to note the most massive SF galaxies $\left(M_{*} \sim 1-3 \times 10^{11} M_{\odot}\right)$ that lie on the locus are missing in our sample, but constitute a considerable fraction in the sample studied by Elbaz et al. (2007). In our sample galaxies with masses above $\sim 10^{11} M_{\odot}$ deviate from the linear relation and have lower SFRs. These massive red galaxies mostly host SNe Ia. In general, the distribution of the galaxies in our sample is very similar to Fig. 17 in Brinchmann et al. (2004), which represents a larger low-redshift sample.

The fact that the galaxies of the different SN types have on average different masses but the same SFRs directly and indirectly affects other galaxy properties. The specific SFR (sSFR $\equiv$ $\mathrm{SFR} / \mathrm{mass}$ ) of SN Ia hosts is lower than CC SNe. Most of the stellar mass of a galaxy is locked in its old stellar populations. Therefore we also expect differences in the compressed population vectors and the mean stellar age. In Fig. 9 the compressed young $x_{\mathrm{Y}}$ and old $x_{\mathrm{O}}$ population vectors and the mean lightweighted stellar age are shown. As expected, all three plots indicate that SN Ia hosts contain older SPs than the hosts of CC SNe. Compared with the SN II hosts, the hosts of $\mathrm{SNe} \mathrm{Ib} / \mathrm{c}$ have fewer old SPs and a slightly younger mean stellar age. This is probably because the $\mathrm{SN} \mathrm{Ib/c} \mathrm{hosts} \mathrm{have} \mathrm{slightly} \mathrm{lower} \mathrm{mass} \mathrm{than}$ those of SNe II.

The $\mathrm{H} \alpha$ line luminosity is an indicator of the ongoing SFR. The $\mathrm{H} \alpha$ equivalent width (EW), on the other hand, measures how strong the line is compared with the continuum. The continuum light is dominated by old stars, which also contain most of the galaxy stellar mass. Thus, it may be expected that $\mathrm{H} \alpha \mathrm{EW}$ is different in the hosts of the different SN types. Moreover, it is heavily correlated to the sSFR, as shown in Sánchez et al. (2013), who used HII regions measured from CALIFA data. $\mathrm{H} \alpha$ EW can be thought of as an indicator of the strength of the ongoing SFR compared with the past SFR. The cumulative distribution of $\mathrm{H} \alpha$ EW shows in Fig. 10 that SN Ia hosts have lower $\mathrm{H} \alpha$ EWs than CC SN hosts. Among CC SNe, SN Ib/c hosts have on average slightly larger EW than SN II hosts. SN Ib/c also lack hosts with $\mathrm{H} \alpha E W<10 \AA$. 
L. Galbany et al.: Nearby supernova host galaxies from the CALIFA Survey. I.
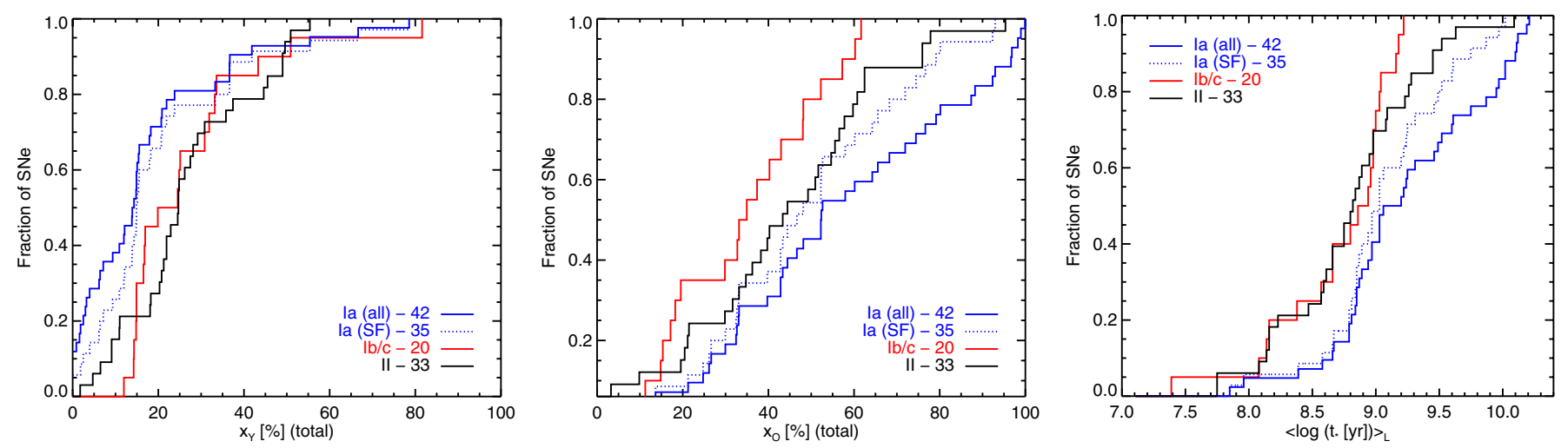

Fig. 9. Cumulative distributions of the contribution of young (left) and old (middle) SPs and the mean light-weighted logarithm of the stellar population age (right).

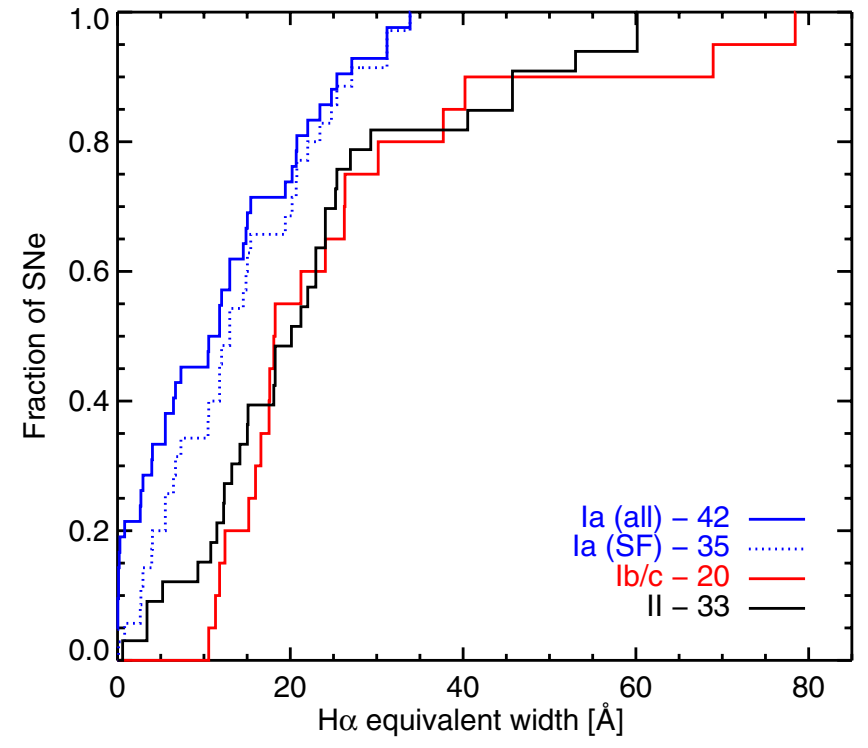

Fig. 10. Cumulative distributions of $\mathrm{H} \alpha$ equivalent width measured from the total galaxy spectra.

Finally, Fig. 11 shows the position of the galaxy centers on the BPT diagram. A considerable fraction of all galaxies appears to host AGN, but no AGN is strong enough to significantly affect the total galaxy spectrum. This shows a potential problem for obtaining spectroscopy of galaxies at high-z, and will be analyzed elsewhere. There is a difference in the fraction of AGNs between the hosts of the three SN types. About $50 \%( \pm 8 \%), 30 \%( \pm 8 \%)$, and $20 \%( \pm 9 \%)$ of the hosts of SN Ia, II, and Ib/c, respectively, host AGNs.

\subsection{Galaxy properties at the SN positions}

\subsubsection{Local versus global properties}

The cumulative distributions of the local SFR density ( $\Sigma$ SFR) at the SN position are shown in Fig. 12. The star formation is spiral galaxies is confined to a thin disk in the galactic plane. Therefore the SFRs measured in the individual spaxels need to be corrected for the galaxy inclination to obtain the true $\Sigma$ SFR. From Fig. 12 one can see that on average the local $\Sigma$ SFR is different for the three $\mathrm{SN}$ types. The mean values of $\log (\Sigma \mathrm{SFR})$ are $-2.44,-2.05$, and -1.80 for SN Ia, SN II, and SN Ib/c, respectively. In comparison, the mean total SFR in the SF galaxies

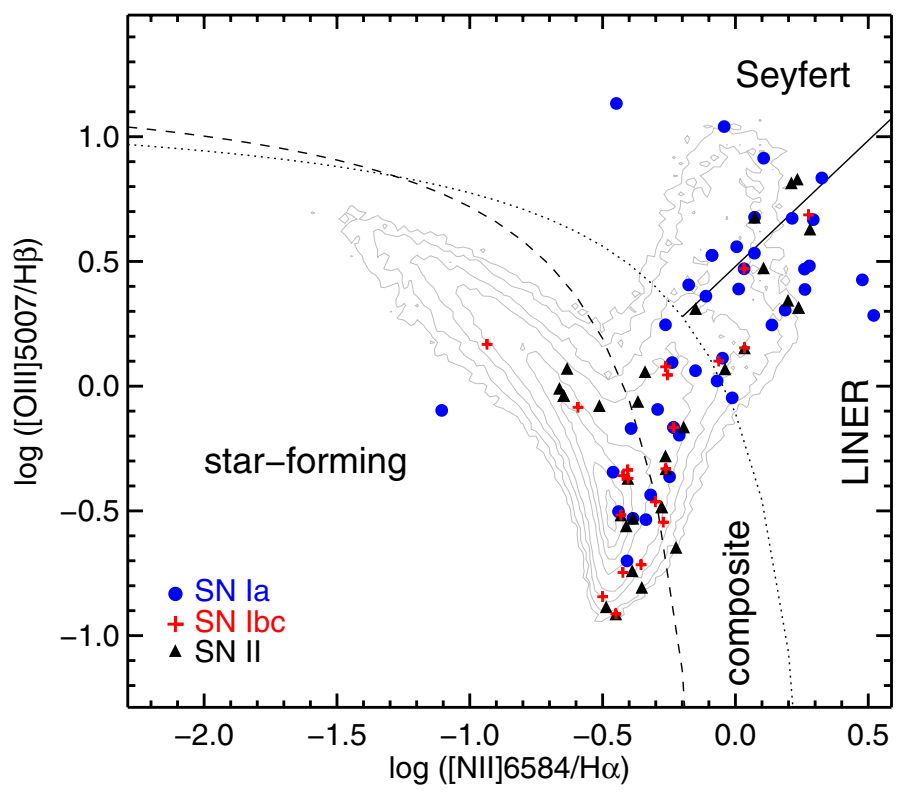

Fig. 11. BPT diagram of the central galaxy spaxel. The contours show the density of SDSS emission line galaxies.

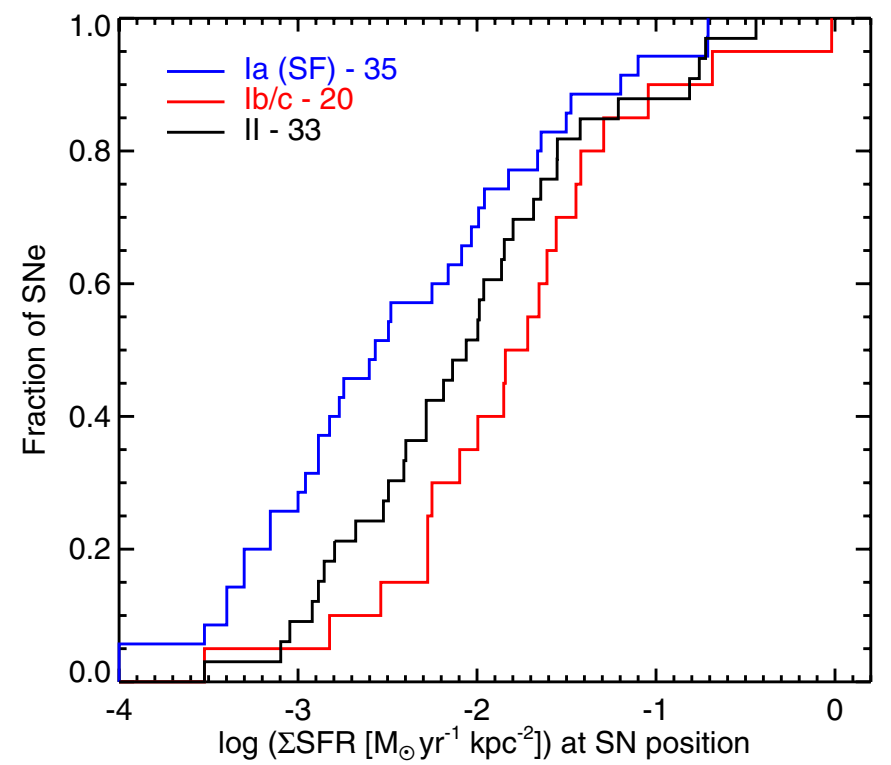

Fig. 12. Cumulative distributions of $\Sigma$ SFR at $\mathrm{SN}$ positions for the three SN types. 

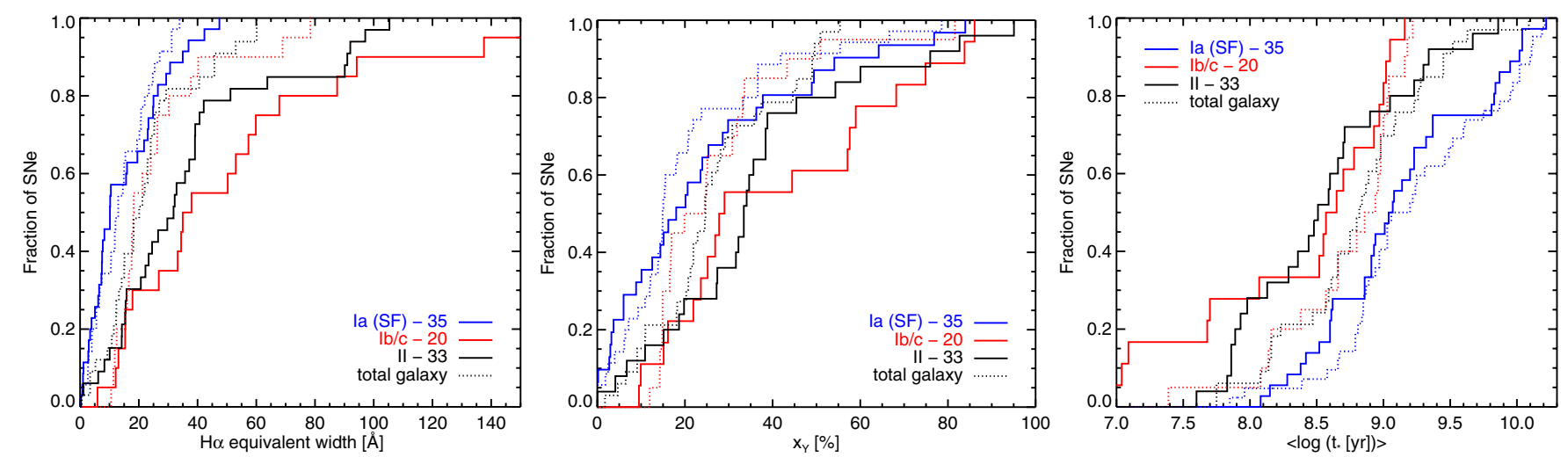

Fig. 13. Cumulative distributions of the $\mathrm{H} \alpha$ equivalent width (left), the contribution of young SPs (middle), and the mean light-weighted logarithm of the stellar population age (right). The solid lines show the CDs of the quantities at the SN position and the dotted lines from the total spectra.

is very similar between the three $\mathrm{SN}$ types. In galaxies with a similar total SFR the different SN types apparently tend to explode in regions with different local SFRs, higher for $\mathrm{SNe} \mathrm{Ib} / \mathrm{c}$, followed by SN II and SN Ia.

The differences between the average $\log (\Sigma \mathrm{SFR})$ for the three $\mathrm{SN}$ types are on the same order as many of the uncertainties of the individual measurements, which raises the question of how significant these differences are. The standard errors of the mean (not given in Table 5) are $\sim 0.15$ dex. Thus the difference between $\mathrm{SNe}$ Ia and II is significant at a level of $\sim 2 \sigma$ and between SNe II and $\mathrm{Ib} / \mathrm{c}$ at a level of $\sim 1 \sigma$.

The analysis of the other indicators we studied also corroborates the result of increasing the difference between the three $\mathrm{SN}$ types when the galaxy properties at the SN position are compared with those from the total spectra. In Fig.13 we compare the local versus total cumulative distributions of $\mathrm{H} \alpha \mathrm{EW}$, mean light-weighted stellar age, and $x_{\mathrm{Y}}$. We present the results only for the SF galaxies, but qualitatively similar trends are also observed when passive galaxies are included in the SN Ia sample.

The cumulative distributions of the local and the global quantities for the SN Ia sample are very similar. This suggests that $\mathrm{SNe}$ Ia do not tend to explode in regions with specific properties, but are rather randomly distributed in the galaxies. If it were otherwise, there should have been a systematic difference between the local and global properties. In contrast, the results for CC SNe show such systematic differences. The CC SNe appear to explode at locations associated with stellar populations younger than the galaxy average. There are also tentative indications that $\mathrm{SN} \mathrm{Ib/c} \mathrm{prefer} \mathrm{regions} \mathrm{with} \mathrm{younger} \mathrm{stellar} \mathrm{populations}$ than SN II.

\subsubsection{Pixel statistics}

Anderson et al. (2012) used the statistical method described in James \& Anderson (2006), which they named normalized cumulative rank pixel function (NCR), to study the correlation of different $\mathrm{SN}$ types to the star formation in the galaxy. The construction of the NCR function in given galaxy basically consists of sorting the $\mathrm{H} \alpha$ flux values in increasing order form the cumulative distribution, and normalize this to the total emission of the galaxy. This associates each pixel with an NCR value between 0 and 1 , where 1 is the brightest pixel and 0 the pixels without emission. By collecting all the NCR values of the pixels that contain SN explosions, one can form the NCR distribution of each SN type. Assuming that the $\mathrm{H} \alpha$ emission scales by the number of stars that are formed (Kennicutt et al. 1994),

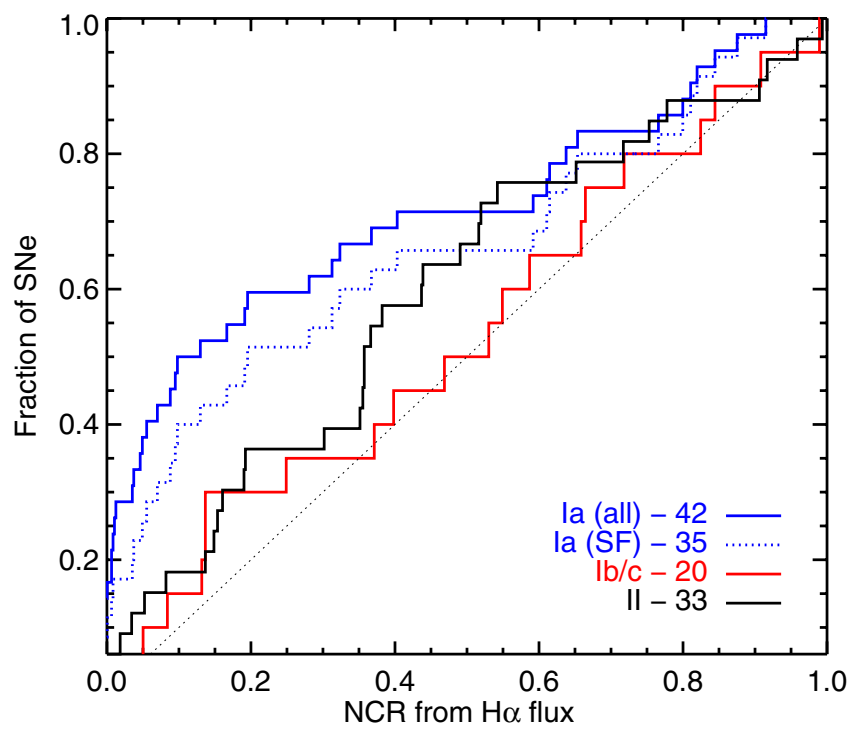

Fig. 14. NCR from $\mathrm{H} \alpha$ flux maps for the $3 \mathrm{SN}$ types. The diagonal line represent a cumulative distribution perfectly describing the star formation in the galaxy. We excluded the $7 \mathrm{SNe}$ Ia in galaxies without emission lines to properly compare the distributions of each SN type in similar galaxies.

a flat NCR distribution (or diagonal cumulative NCR distribution) with a mean value of 0.5 would mean that this type of SN accurately follows the stars that are formed and mapped by that particular SF tracer. In a typical galaxy, more regions have a low SFR, therefore the NCR values will be predominantly low and the cumulative distribution will lie above the diagonal if certain SN type explode at random locations. On the other hand, if SNe explode predominantly in locations with high SFR, the line will be below the diagonal.

We computed the NCR cumulative distributions with our extinction-corrected $\mathrm{H} \alpha$ flux 2D maps. To properly compare the distributions for each SN type, we only considered SNe Ia that exploded in the SF galaxies. Figure 14 shows the resulting NCR distributions for each sub-sample. The SN Ibc sample is closest to a flat distribution $(\langle N C R\rangle=0.47)$ and closely tracing the star formation in the galaxy. The following group associated with SF is SN II (0.40), followed by SN Ia (0.34-0.28). This result supports the findings of Anderson et al. (2012) that $\mathrm{SN} \mathrm{Ib/c} \mathrm{are} \mathrm{most} \mathrm{closely} \mathrm{associated} \mathrm{with} \mathrm{star} \mathrm{formation,} \mathrm{fol-}$ lowed by SN II and SN Ia. This also agrees with our results from the previous section. 

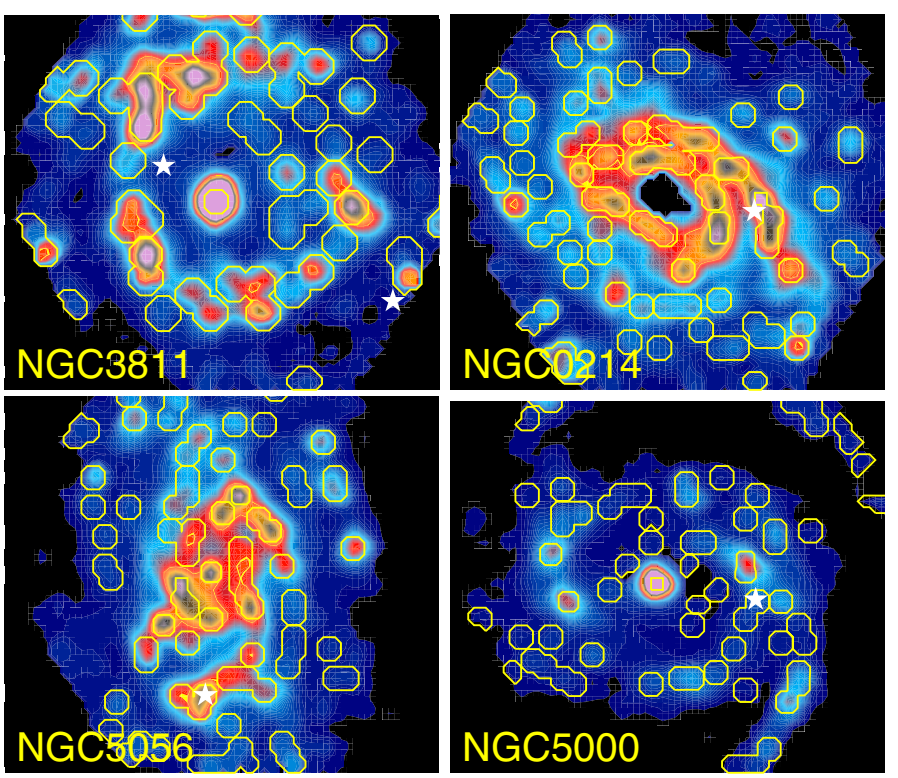

Fig. 15. Example of HiI regions determined by HIIEXPLORER. The white star shows the SNe positions. Galaxies are ordered from lower to higher redshift. This shows the effect of placing an upper limit on the extension of the $\mathrm{H}$ II clump that never exceeds $1 \mathrm{kpc}$ diameter.

The two-sample K-S tests between the NCR distributions indicates that $\mathrm{SN} \mathrm{Ib/c}$ and $\mathrm{SNe}$ II samples come from similar underlying distributions with a probability of 0.8 . The SN Ia distribution is significantly different from the two CC SNe distributions however, the probability that it came from the same distribution as the $\mathrm{SNe} \mathrm{Ib} / \mathrm{c}$ is 0.15 . The KS tests between each distribution and the diagonal distribution support the fact that $\mathrm{SNe} \mathrm{Ibc}(0.65)$ follows the SF distribution better than the SNe II (0.09) and SNe Ia (1.59e-03-2.85e-06) distributions.

\subsubsection{H II clump detection from $\mathrm{H} \alpha$ map}

The distance from the SN position to the nearest SF region within the host galaxy can also be used to study the association of different $\mathrm{SN}$ types with star formation. In our analysis, the $\mathrm{SF}$ regions were selected with HIIEXPLORER ${ }^{11}$, a package that detects $\mathrm{H}$ II clumps (aggregation of $\mathrm{H}$ II regions) in $\mathrm{H} \alpha$ intensity maps (Sánchez et al. 2012b). We used our extinction-corrected $\mathrm{H} \alpha$ maps of the SF galaxies as input. The pixels corresponding to the AGN contribution were also removed from the maps. Starting from the brightest pixel in the map, the code aggregates the adjacent pixels until all pixels with a flux higher than $10 \%$ of the peak flux of the region and within 500 pc or 3.5 pixels from the center have been accumulated. The distance limit takes into account the typical size of $\mathrm{H}$ II regions of a few hundreds of parsecs (e.g., González Delgado \& Pérez 1997; López et al. 2011). The upper limit of 3.5 pixels helps to increase the spatial resolution in the galaxies at low redshifts. The selected region is masked, and the code iterates until no peak with a flux exceeding the median $\mathrm{H} \alpha$ emission flux of the galaxy is left.

Figure 15 presents some examples of the H II clump determination. We do not select individual $\mathrm{H}$ II regions with this procedure. Indeed, the physical scale of a real H II region can be significantly smaller than the pixel size. As a result, the H II clumps would contain more than one $\mathrm{H}$ II region, especially in more distant galaxies. Mast et al. (2014) studied the loss of resolution

\footnotetext{
${ }^{11}$ http://www.caha.es/sanchez/HII_explorer/
}

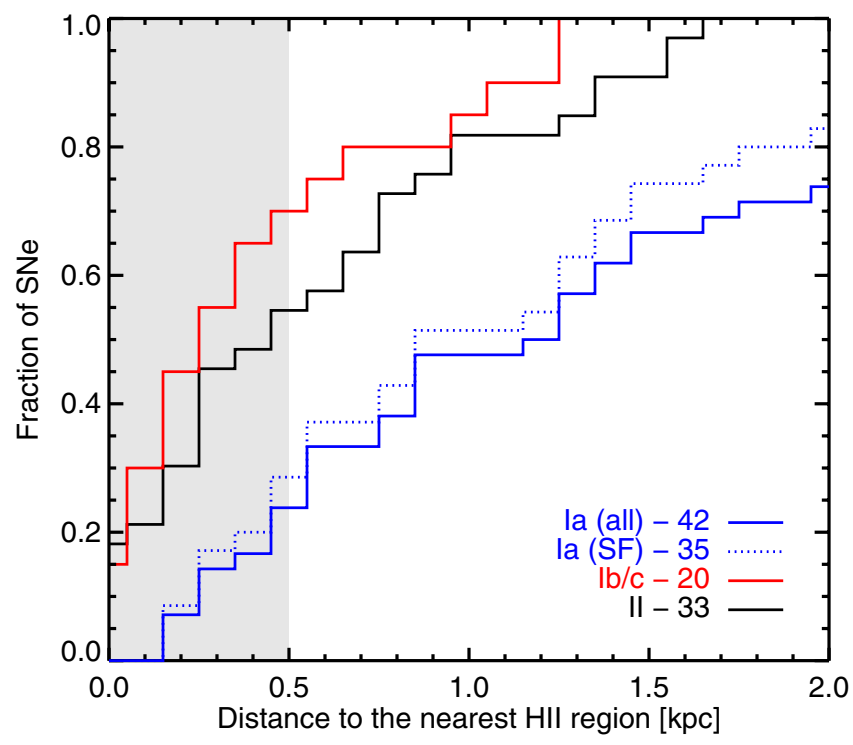

Fig. 16. Cumulative distributions of the distances to the nearest $\mathrm{H}$ II region in bins of $100 \mathrm{pc}$. The shadowed region represents the larger radius of an $\mathrm{H}$ II region assumed in this study.

in IFS using nearby galaxies observed by PINGS. Some of these galaxies were simulated at higher redshifts to match the characteristics and resolution of the galaxies observed by the CALIFA survey. The authors conclude for the H II region selection, that at $z \sim 0.02$ the H II clumps can on average contain from 1 to 6 of the H II regions obtained from the original data at $z \sim 0.001$. Another caveat is that this procedure tends to select regions with similar sizes, although real $\mathrm{H}$ II regions have different sizes. Furthermore, the SN progenitor might be formed in other $\mathrm{H}$ II regions that are not detected by this method. Considering this as our best approximation because of the resolution of the data, we refer to our segregated regions as H II regions throughout this paper.

After we determined the $\mathrm{H}$ II regions in all galaxies, we calculated the distance between the SN and the center of the nearest $\mathrm{H}$ II region on the galaxy disk (deprojected distance). In Fig. 16 all distributions are plotted in bins of $100 \mathrm{pc}$, the estimated average error of all distance measurements. We find a sequence ranging from $\mathrm{SNe} \mathrm{Ib} / \mathrm{c}, \mathrm{SNe} \mathrm{II}$, and finally $\mathrm{SNe} \mathrm{Ia}$, which are less associated with $\mathrm{H}$ II regions. The K-S tests show that both CC SNe distributions are independent of SNe Ia distribution, but they can come from similar populations.

Note that these are distances to the center of the H II region. A star that was born at the border of a large $\mathrm{H}$ II region of $500 \mathrm{pc}$ of radius may have traveled $\sim 600 \mathrm{pc}$ before its death (Hammer et al. 2006; Anderson et al. 2010). In that case, we would calculate $1.1 \mathrm{kpc}$ as the distance from the $\mathrm{SN}$ to the center of its $\mathrm{H}$ II region. $70 \%( \pm 10 \%)$ of $\mathrm{SNe} \mathrm{Ib} / \mathrm{c}$ and $55 \%( \pm 9 \%)$ of $\mathrm{SNe}$ II are inside or closely associated to SF regions $(<0.5 \mathrm{kpc})$, while the position of SNe Ia are less correlated to SF region, with fewer than $25 \%( \pm 7 \%)$ of them falling nearby/inside a SF clump.

\section{Discussion}

\subsection{Correlation to star-forming regions}

We have performed several analyses to probe the degree of correlation of the three main SN types with star formation. In all of them, we found a sequence in which stripped-envelope 
$\mathrm{SNe} \mathrm{Ibc} / \mathrm{IIb}$ had the highest degree of correlation and $\mathrm{SNe} \mathrm{Ia}$ had the lowest correlation.

As in Anderson et al. (2012), we found that $\mathrm{SNe} \mathrm{Ib/c} \mathrm{are}$ more closely associated with H II regions than SNe II, which is consistent with the latter SN type coming from less massive progenitors. In a SF region the most massive stars generate the bulk of the UV radiation, which ionizes the circumstellar hydrogen and produces a bright H II region (Kennicutt 1998a; Gogarten et al. 2009). The massive stars have short lifetimes and explode first, close to their birth place, because they have not had enough time to travel away. The removal of the massive stars from the $\mathrm{H}$ II region reduces the UV radiation and hence the intensity of the $\mathrm{H} \alpha$ emission also decreases. Therefore, when the less massive stars explode as SNe later on, they do so in an environment with lower $\mathrm{H} \alpha$ emission. In addition, the explosion of the most massive stars in the $\mathrm{H}$ II region drives the removal of gas from it, which also contributes to the decrease of the $\mathrm{H} \alpha$ emission. The explosion also causes high peculiar motions of the surrounding less massive stars (Eldridge et al. 2011), and they will explode farther away of the H II region center. For these reasons, high-mass SN progenitors probably trace the ongoing star formation better than low-mass progenitors. Moreover, as discussed by Crowther (2013), most H II regions detected in ground-based imaging are not classical compact $\mathrm{H}$ II, but rather giant HII regions, which tend to have a complex structure and a relatively long duty cycle of $\sim 20$ Myr. For this reason, the association of $\mathrm{SNe}$ with nearby $\mathrm{H}$ II regions provides relatively weak constraints on the CC SN progenitor masses.

The SNe Ia are the class least associated to SF regions. This is expected because SNe Ia are observed in elliptical galaxies, which contain only old SPs (Kehrig et al. 2012). It can almost certainly be excluded that SN Ia in elliptical galaxies come from young progenitors from weak residual star formation. According to the nearby SN rates estimated by Maoz et al. (2011), a young star burst $(<420 \mathrm{Myr})$ produces ten times more CC SNe than $\mathrm{SNe}$ Ia. Although a few CC SNe have been observed in elliptical galaxies that show signs of star formation from UV observations (e.g., Hakobyan et al. 2008; Suh et al. 2011), they are much rarer than SNe Ia. Therefore, at least part of SNe Ia probably come from old SPs, as demonstrated by Maoz et al. (2011, 2010). $\mathrm{SNe}$ Ia observed in spiral galaxies might in principle come from their old SPs. However, many recent studies have shown that at least part of the SNe Ia are probably produced by SPs as young as 100-300 Myr (Mannucci et al. 2005; Scannapieco \& Bildsten 2005; Sullivan et al. 2006; Maoz et al. 2010; Brandt et al. 2010; Maoz \& Badenes 2010). Thus, the SN Ia population in spiral galaxies is most likely a mixture of events from old and young progenitors, as imprinted in their delay-time distribution (DTD, the time between the progenitor formation and the SN explosion), and we do not expect to see a clear correlation to star formation.

\subsection{Global galaxy properties}

The analysis of the total galaxy properties showed that in our sample the SN Ia hosts are more massive than the SN II hosts. The mean diffence is $\sim 0.3$ dex if only spiral galaxies are taken into account for SNe Ia or $\sim 0.4$ dex when the whole sample is used. At the same time, the total ongoing SFR in the SN Ia and $\mathrm{CC} \mathrm{SNe}$ is on average the same. As we discussed in Sect. 4.1, these two parameters directly and indirectly affect other galaxy properties.

The apparent lack of nearby SNe Ia in hosts with masses lower than $\sim 10^{10} M_{\odot}$ is usually attributed to the fact that nearby

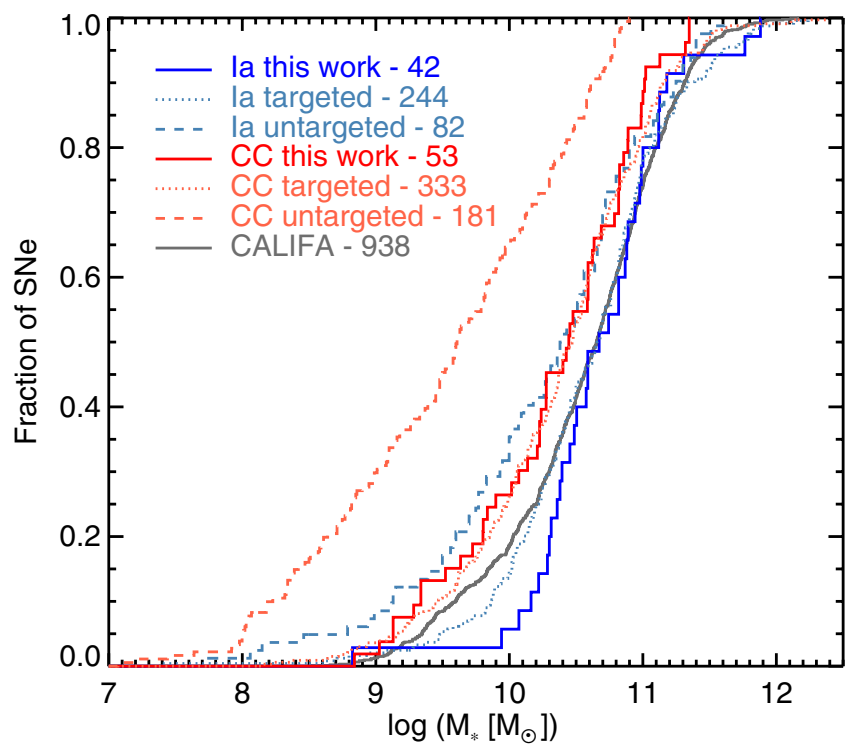

Fig. 17. Cumulative distributions of the host galaxy masses of various SN Ia and CC SNe samples (see the text for details).

SNe Ia are discovered by search programs that target bright massive galaxies. From the untargeted SN surveys at high redshift it is known that $\mathrm{SNe}$ Ia do explode in low-mass galaxies (e.g., Sullivan et al. 2006), and now The Supernova Factory and the Palomar Transient Factory (PTF) have shown this to the case at low redshifts as well (Childress et al. 2013; Pan et al. 2014). On the other hand, the targeted searches find many CC SNe in galaxies with masses lower than $\sim 10^{10} M_{\odot}$. Therefore, the targeted searches also sample low-mass galaxies, but very few SNe Ia are found in them.

To investigate whether the difference between the masses of SN Ia and CC SN hosts is specific to our sample, we compared our results with the host masses of the SNe discovered by other surveys. Figure 17 shows the CDFs of the masses of our SN Ia and CC SN hosts with the untargeted SN Ia hosts from PTF (Pan et al. 2014), the untargeted CC SNe hosts from PTF (Stoll et al. 2013) and from Kelly \& Kirshner (2012) compiled from several different surveys, as well as the targeted CC SN and SN Ia galaxy samples of Kelly \& Kirshner (2012) and Neill et al. (2009). The SN Ia and CC SN samples have the same redshift range $z \sim 0.001-0.08$ with the CC SNe skewed toward the lower and $\mathrm{SNe}$ Ia toward the higher redshifts. We show the full CALIFA mother sample, which lacks lower mass galaxies because they were selected by size to fulfill the instrument FoV, thus missing dwarf galaxies.

Figure 17 clearly shows that the targeted surveys are biased toward massive galaxies (a KS between our sample and those of the literature yielded Ia targ. 0.48, CC targ. 0.83, Ia untarg. 9.5e-03, CC untarg. 2.3e-06). Although the untargeted surveys discover more $\mathrm{SNe}$ in hosts with masses lower than $\sim 10^{10} M_{\odot}$, the difference between the mean masses of CC SN and $\mathrm{SNe}$ Ia hosts increases to $0.6-0.8 \mathrm{dex}$. The mass distribution of the CC SN hosts in our sample matches the targeted sample compiled from the literature very well. We compared the masses of SNe Ia hosts from targeted surveys of our sample with the larger sample of Neill et al. (2009). The distributions are very similar at the high-mass end, but our sample is slightly biased toward higher mass at the low-mass end.

The analysis of the host galaxy masses indicates that with increasing galaxy mass the production of SN Ia with respect to 
$\mathrm{CC} \mathrm{SNe} \mathrm{increases.} \mathrm{This} \mathrm{can} \mathrm{be} \mathrm{understood} \mathrm{as} \mathrm{a} \mathrm{result} \mathrm{of} \mathrm{the} \mathrm{dif-}$ ferences between the DTD of the two SN types. CC SNe explode within $\sim 40 \mathrm{Myr}$ from the onset of the star formation. On the other hand, there is growing evidence that DTD of SNe Ia is a continuous function between $100 \mathrm{Myr}$ and $11 \mathrm{Gyr}$ with a form close to DTD $\propto t^{-1}$ (Maoz et al. 2010, 2011). This means that about half of SNe Ia come from progenitors older than about 1 Gyr. The ongoing SFR in galaxies with mass $M_{*} \leq 10^{11} M_{\odot}$ is a power function of the mass with exponent 0.77 (Elbaz et al. 2007). This implies that the more massive galaxies contain larger fractions of old SPs than the lower mass galaxies. Therefore with increasing galaxy mass the ratio of SN Ia to CC SNe will increase. This effect will be even more pronounced for the most massive galaxies with $M_{*} \geq 10^{11} M_{\odot}$, for which the SFR saturates.

The code STARLIGHT provides not only the current mass of the SPs in the galaxies, but also their initial mass. This can be used to compute the total mass ever converted into stars as a function of time (Pérez et al. 2013; González Delgado et al. 2014a). For the galaxies in our sample we computed the total mass converted into stars in the time intervals $0-0.42 \mathrm{Gyr}$, 0.42-2.4 Gyr, and >2.4 Gyr, which correspond to the young, intermediate, and old SPs used by Maoz et al. (2010) and Maoz et al. (2012) in their recovery of the DTD. On average, the CC SN hosts formed $2.9 \times 10^{7} M_{\odot}, 1.2 \times 10^{9} M_{\odot}$, and $3.4 \times 10^{10} M_{\odot}$ in these intervals $(0.1 \%, 3.4 \%$, and $96.5 \%$ ot the total mass formed, respectively), and the SF SN Ia hosts $3.3 \times 10^{7} M_{\odot}, 1.6 \times 10^{9} M_{\odot}$, and $7.9 \times 10^{10} M_{\odot}(0.04 \%, 2.01 \%$, and $97.95 \%$ ). With the SN Ia rates of Maoz et al. (2012) we estimate that on average the SN Ia hosts in our sample should have produced about twice more SNe Ia than the CC SN hosts. Even though the young SPs of a galaxy produce many more $\mathrm{SNe}$ Ia than the old SPs per unit mass converted into stars, the recent star formation in most disk galaxies is much less intense than in the past, and most of their stellar mass is locked into the old SPs. In a galaxy with a SFH similar to that of the galaxies in our sample, only $\sim 2-5 \%$ of the SNe Ia observed today come from the young SPs $(<0.42 \mathrm{Gyr})$ and $\sim 25 \%$ from the intermediate $(0.42-2.4 \mathrm{Gyr})$. For this reason, the SN Ia rate in these galaxies is nearly proportional to their masses, which might explain why the SN Ia hosts are more massive than those of CC SNe. Moreover, the mass formed during the last 420 Myr follows nearly the same distribution for our SN Ia and CC SN hosts, which is consistent with our previous estimate from $\mathrm{H} \alpha$ flux: the hosts of the two SN types have on average the same ongoing SFR.

Eight galaxies in our sample have masses lower than $3.3 \times 10^{9} M_{\odot}$ and an average mass of $1.6 \times 10^{9} M_{\odot}$. All these galaxies belong to the group of blue galaxies (Fig. 2 lower panel). From the SFHs recovered with STARLIGHT we find that $1.3 \times 10^{7} M_{\odot}, 4.9 \times 10^{8} M_{\odot}$ and $1.5 \times 10^{9} M_{\odot}$ stars $(0.65 \%$, $24.46 \%, 74.89 \%$ ) formed in the three age bins. Taking as a reference the massive SF SN Ia hosts (mean mass $4.7 \times 10^{10} M_{\odot}$ ), most of which belong to the red group, the eight low-mass galaxies formed their stars over a longer time and contain a larger fraction of young SPs. By repeating the calculations from the previous paragraph, we find that these galaxies probably produce ten times fewer SNe Ia than the massive SN Ia hosts. However, the ratio of the mean masses is about 30 . Therefore the low massive galaxies produce at present three times more $\mathrm{SNe}$ Ia per unit mass, and the difference comes from the increased fraction of SNe Ia from the younger progenitors: $\sim 10 \%$ from the young SPs $(<0.42 \mathrm{Gyr})$ and $\sim 75 \%$ from the intermediate-age SPs $(0.42-2.4$ Gyr).

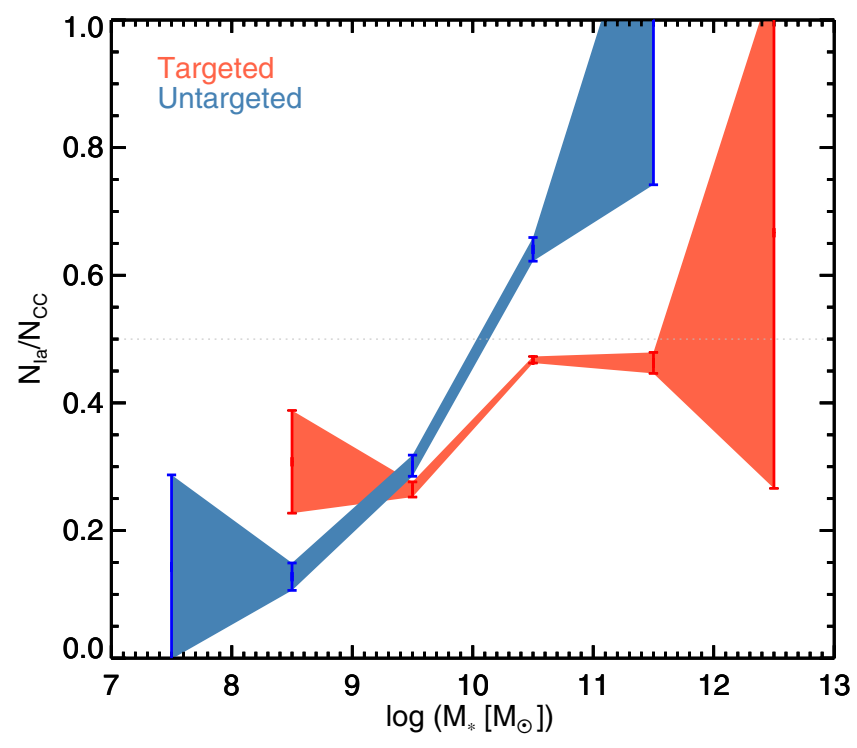

Fig. 18. Ratio between the number of SNe Ia and CC SNe in the compilation of targeted and untargeted galaxies in 1 dex galaxy mass bin.

The picture should be much simpler for the CC SNe. They come from massive stars with mass $M \geq 8 M_{\odot}$, which explode within $\sim 40 \mathrm{Myr}$ after the star formation, and the rate of CC SNe should be proportional to the ongoing SFR. The average SFR of the massive SN Ia hosts and the low-mass galaxy groups is $1.51 M_{\odot} \mathrm{yr}^{-1}$ and $0.51 M_{\odot} \mathrm{yr}^{-1}$, which implies that the low-mass galaxies should produce three times fewer CC SNe than the massive galaxies. They produced ten times fewer $\mathrm{SNe} \mathrm{Ia}$, and therefore the ratio of $\mathrm{CC} \mathrm{SNe}$ to $\mathrm{SNe}$ Ia is expected to increase with decreasing galaxy mass. Thus, we expect to discover SNe Ia preferably in high-mass galaxies and CC SNe in low-mass galaxies. However, various biases can influence the $\mathrm{SN}$ discoveries. Most CC SNe are less luminous by at least 1-2 mag than SNe Ia. Thus, CC SNe should be easier to discover in low-mass, low surface brightness galaxies. A potential manifestation of this effect can be seen in Fig. 17. No untargeted SN searches seem to have discovered $\mathrm{CC} \mathrm{SNe}$ in galaxies with masses higher than $\sim 10^{11} M_{\odot}$, while PTF clearly discovers SNe Ia in such galaxies. Figure 18 shows the ratio of $\mathrm{SNe}$ Ia to $\mathrm{CC}$ SNe in different galaxy mass bins for the compiled sample detailed above, where the targeted SNe Ia sample was restricted to the same redshift range as the targeted CC SN sample $(z<0.023)$ to allow a proper comparison. It clearly indicates that the $\mathrm{SNe}$ Ia contribute more when the mass of the host galaxy increases. This result agrees with previous works by Boissier \& Prantzos (2009), Foley \& Mandel (2013), and Hakobyan et al. (2014) who used absolute $B$ magnitude and morphology as a proxy for the stellar mass.

\subsection{Local versus integrated}

The spectra of high-redshift galaxies are usually obtained with long-slit or fixed aperture fibers that integrate the light of the whole (or nearly the whole) galaxy. The SN Ia community has long been searching for additional parameters that can improve SNe Ia as a standard candle. Kelly et al. (2010), Sullivan et al. (2010), and Lampeitl et al. (2010) were among the first to suggest that the residuals from the best-fit Hubble line (Hubble residuals, or HRs from now on) correlate with the SN host stellar mass. Furthermore, Sullivan et al. (2010) proposed to incorporate two different absolute peak magnitudes into the 

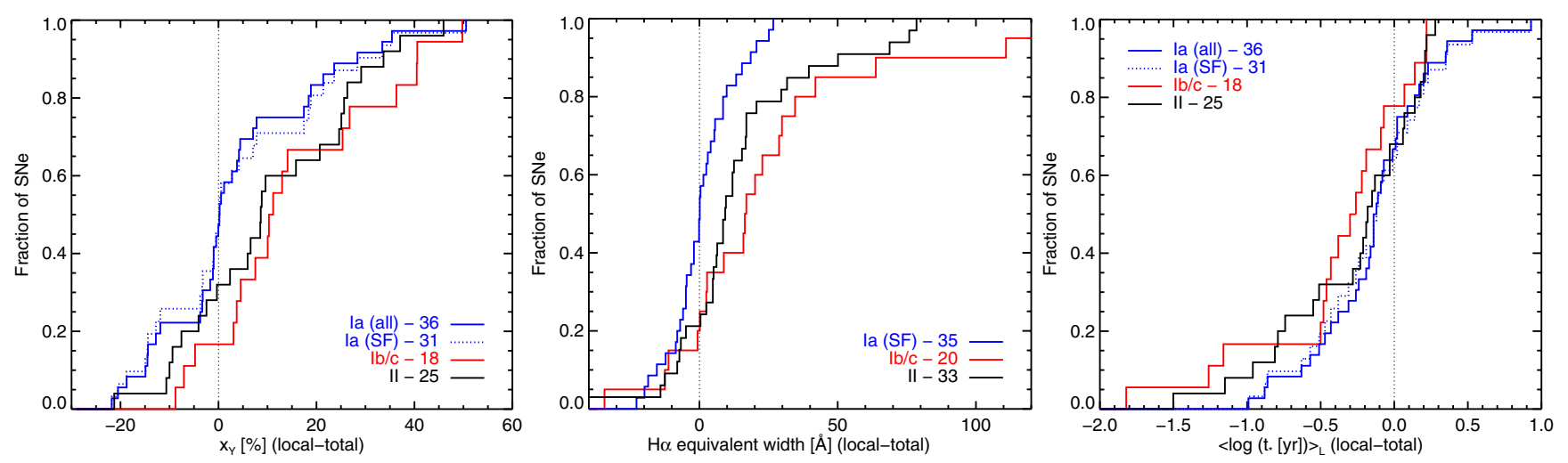

Fig. 19. Cumulative distributions of the difference between the local and global properties. The contribution of young SPs (left), H $\alpha$ equivalent width (middle), and the mean light-weighted logarithm of the stellar population age (right).

cosmological SN Ia analyses for SNe in hosts with masses lower or higher than $10^{10} M_{\odot}$. The cause of this apparent correlation is unclear. Metallicity, SFR, SPs age, and other galaxy parameters are known to correlate with the galaxy mass. For example, our analysis of the low-mass galaxy group and the massive SN Ia hosts showed that the low-mass galaxies formed most of their stars later than the massive ones and so contain more younger SPs. On one hand, the parameters that are candidates to drive the correlation can also affect the properties of the WD population that gives rise to $\mathrm{SNe}$ Ia. On the other hand, many theoretical investigations have shown that the properties of the exploding WDs can affect the amount of ${ }^{56} \mathrm{Ni}$ that is synthesized in the explosion and hence the SN luminosity (see, e.g., Timmes et al. 2003; Röpke et al. 2006; Howell et al. 2009; Bravo et al. 2010, and references therein).

From our IFS data we see that all these parameters are nonuniformly distributed in the galaxies, most often showing radial gradients (see, e.g. S12). This poses the question whether using the local values of these parameters measured at the SN position instead of the total values can additionally improve this. While the spatially resolved IFU spectroscopy is best suited for testing this, we are still not in a position to do so. One of the problems is that we have IFU observations only of massive SN Ia hosts that span a limited mass range. On the other hand, only a few of the $\mathrm{SNe}$ observed in these galaxies have published light curves that are good enough to derive the SN peak magnitudes. With the sample at hand we can test how different the local and total quantities are. In the future, if a correlation with the local properties is found, we can asses the errors we will make when the global values are used instead. This may be relevant, for example, for $\mathrm{SNe}$ at high-redshft, for which only the total galaxy spectrum can be measured.

Figure 19 shows the CDFs of the difference between the local and total values of several galaxy parameters that may be of interest (only for the SF hosts of SNe Ia). These parameters are sensitive to the presence of young stellar SPs $-x_{\mathrm{Y}}$, mean $\log ($ age $)$, and $\mathrm{H} \alpha \mathrm{EW}$ - indicate that the CC SNe in our sample explode in regions with more young stars than the galaxy mean. This difference supports the idea that the total value cannot be used as a proxy for the local value (Modjaz et al. 2008). On the other hand, the mean difference between the local and total values for SNe Ia is much closer to zero: $1 \pm 12 \AA,-4 \pm 18 \%$, and $-0.14 \pm 0.42$ dex for $\mathrm{H} \alpha \mathrm{EW}, x_{\mathrm{Y}}$, and $\log ($ age $)$, respectively. This means that $\mathrm{SNe}$ Ia do not tend to explode in regions with some characteristic SPs, but are instead randomly distributed round the value inferred from the total galaxy spectrum (although the distributions are slightly skewed). Thus, the value derived from the total spectrum can be used to obtain an estimate, albeit not very accurate in some cases, for the range of values to expect at the $\mathrm{SN}$ position. The uncertainty is given by the standard deviation of the distributions shown in Fig. 19. The difference between $\log (\Sigma \mathrm{SFR})$ and $\log ($ total SFR) for SN Ia, Ib/c, and II are -2.5 , -2.0 , and -2.2 dex, respectively, all with $1 \sigma$ scatter $\sim 0.75$ dex.

\section{Conclusions}

We analysized IFU spectroscopy of 81 galaxies that hosted $42 \mathrm{SNe} \mathrm{Ia}, 20 \mathrm{SNe} \mathrm{Ib} / \mathrm{c}$, and $33 \mathrm{SNe}$ II (95 in total). In this first study of a series we focused on the galaxy properties that are related to the star formation and the galaxy star formation histories. The observations were obtained from the CALIFA Survey and some other projects using the same instrument. The spatially resolved spectroscopy technique provides much more detailed information about the galaxy properties than integrated spectroscopy or multicolor broad-band imaging because it allows us to obtain 2D maps of many important galaxy characteristics, both for the ionized gas and the stellar populations. In particular, it is possible to accurately obtain the galaxy properties at the projected locations of the SN explosion and compare them with the overall distribution of these properties in the galaxy. The data analysis followed the recipe of S12, and our main findings are summarized below.

By studying various indicators of the ongoing and recent star formation in the galaxies related to the ionized gas and the stellar populations, we confirmed the previously known fact that $\mathrm{SN} \mathrm{Ib/c} \mathrm{are} \mathrm{the} \mathrm{SN} \mathrm{type} \mathrm{most} \mathrm{closely} \mathrm{related} \mathrm{to} \mathrm{SF} \mathrm{regions,} \mathrm{fol-}$ lowed by SN II and finally SNe Ia.

The star formation density at the SN locations forms a sequence that reaches from most intense for $\mathrm{SN} \mathrm{Ib/c} \mathrm{to} \mathrm{weakest} \mathrm{for}$ $\mathrm{SNe}$ Ia, although the differences are only significant at a level of $1-2 \sigma$. At the same time, considering only the SF hosts of $\mathrm{SNe}$ Ia, we found that the total ongoing star formation inferred from $\mathrm{H} \alpha$ emission line luminosity is on average the same for the hosts of the three SN types.

From the $g-r$ vs. $M_{r}$ diagram we found that $>80 \%$ of the $\mathrm{SN}$ Ia hosts are red galaxies, while the hosts of the CC SNe are equally split between red and blue galaxies.

On average, the masses of the SN Ia hosts are higher by $\sim 0.3-0.4$ dex than the masses of the CC SN sample. The SFHs recovered by the full-spectrum fitting with the code STARLIGHT showed that the difference is almost entirely due to the larger fraction of old stellar populations in the SN Ia hosts, even when only the SF SN Ia hosts are considered. 
By using the recent SN Ia delay-time distribution function recovered by Maoz et al. (2012) together with the SFHs we obtained, we showed that the SN Ia hosts in our sample will probably produce twice more SNe Ia than the CC SN hosts. Together with the finding that the hosts of the two SN groups have the same total ongoing SFR, and hence similar CC SN rate, this can explain the mass difference between the SN Ia and CC SN hosts. It also reinforces the finding that at least part of SNe Ia probably originate from very old progenitors.

Our SN sample comes from targeted SN searches, which are known to be biased toward bright, massive galaxies. Using a SN sample discovered by untargeted SN searches from the literature, we showed that the difference between the mean masses of SN Ia and CC SNe hosts not only remains, but increases to $\sim 0.6-0.8$ dex.

We compared the mean SFH derived from the eight least massive galaxies in our sample with mean mass $M_{*} \sim 1.6 \times$ $10^{9} M_{\odot}$ with that of the massive SF hosts of SN Ia with mean mass $M_{*} \sim 4.7 \times 10^{10} M_{\odot}$. The low-mass galaxies formed their stars over a longer time with $0.65 \%, 24.46 \%$, and $74.89 \%$ formed in the intervals $0-0.42 \mathrm{Gyr}, 0.42-2.4 \mathrm{Gyr}$, and $>2.4 \mathrm{Gyr}$, respectively. On the other hand, the massive SN Ia hosts formed $0.04 \%, 2.01 \%$, and $97.95 \%$ of their stars in these intervals. Using the DTD of SNe Ia (Maoz et al. 2012), we estimated that the low-mass galaxies produce about ten times fewer SNe Ia, even though their mass ratio is $\sim 30$. The low-mass galaxies produce about three more SNe Ia per unit mass, and the difference comes from the increased fraction of SNe Ia from younger progenitors $\sim 10 \%$ from the young SPs $(<0.42 \mathrm{Gyr})$ and $\sim 75 \%$ from the intermediate $(0.42-2.4 \mathrm{Gyr})$, compared with only $\sim 3 \%$ and $25 \%$ for the SN Ia hosts.

The mean total ongoing SFR of the high- and low-mass groups is $1.51 M_{\odot} \mathrm{yr}^{-1}$ and $0.51 M_{\odot} \mathrm{yr}^{-1}$, which implies that the low-mass galaxies will probably produce about three times fewer $\mathrm{CC} \mathrm{SNe}$. Therefore the ratio between the number of $\mathrm{CC} \mathrm{SNe}$ and SNe Ia is expected to increase with decreasing galaxy mass, and we expect to discover SNe Ia preferably in high-mass galaxies and $\mathrm{CC} \mathrm{SNe}$ in lower-mass galaxies, as observed. This can be further enhanced by various potential biases that might influence SN discoveries: most CC SNe are by at least 1-2 mag less luminous than $\mathrm{SNe}$ Ia, for example, which makes it harder to detect $\mathrm{CC} \mathrm{SNe}$ in massive, bright galaxies.

$\mathrm{CC} \mathrm{SNe}$ tend to explode at positions with younger stellar populations than the galaxy average. No such bias is observed for SN Ia hosts, and the SP properties at the SN position are one average the same as the global properties. This suggests that $\mathrm{SNe}$ Ia do not tend to explode in regions with specific properties, but are instead randomly distributed in the galaxies. Future studies may find correlations between the local host galaxy properties and the SN Ia light curve parameters that additionally reduce the SN Ia peak magnitudes scatter and therefore sharpen the SN Ia as a standard candle. At very high redshifts only spectra of the total galaxy can be obtained, and our findings suggest that the host galaxy properties inferred from them might be used as an approximation of the local SN Ia properties.

The main long-term goals of this series of works is to study the relation of SN Ia to their local environment to constrain their progenitors and search correlations with the light curve parameters that may improve the SNe Ia as standard candles for measuring distances in the Universe. Currently, only a few galaxies in our sample hosted SNe Ia with published light curves that are good enough to derive the light curve parameters. However, in the future this situation may change. So far, CALIFA has observed $\sim 450$ galaxies, and at the end of the survey it is expected to increase this number to $\sim 600$. Several $\mathrm{SNe}$ have been discovered in galaxies that have previously been observed by CALIFA or by other IFU programs we used. Given the many ongoing large-area SN searches, we expect that this trend will increase in the future. Finally, we suggest all SN searches to include the final galaxy sample observed by CALIFA in their galaxy or survey field lists. This will increase the chances that most of the $\mathrm{SNe}$ that will eventually explode in these galaxies are discovered. This will make it possible to fully exploit the potential of the CALIFA legacy survey for SN progenitor studies.

Acknowledgements. We acknowledge the anonymous referee for her/his helpful comments and suggestions. This work was partly funded by Fundação para a Ciência e a Tecnologia (FCT, Portugal) with the research grant PTDC/CTEAST/112582/2009. V.S. acknowledges financial support from Fundação para a Ciência e a Tecnologia (FCT) under program Ciência 2008. Support for L.G. is partially provided by FCT, by CONICYT through FONDECYT grant 3140566, and from the Ministry of Economy, Development, and Tourism's Millennium Science Initiative through grant IC12009, awarded to The Millennium Institute of Astrophysics (MAS). L.G. thanks Damián Mast, Luzma Montoya, Ana Guijarro and Chandreyee Sengupta for the IFS observations of CALIFA galaxies at Calar Alto Observatory. J.B.-B. and B.G.-L. acknowledge the support from the Plan Nacional de I+D+i (PNAYA) funding programme (AYA201239408-C02-02) of the Spanish Ministry of Economy and Competitiveness (MINECO). J.F.-B. acknowledges support from the Ramon y Cajal Program, grants AYA2010-21322-C03-02 from the Spanish Ministry of Economy and Competitiveness (MINECO). J.F.-B. also acknowledges support from the FP7 Marie Curie Actions of the European Commission, via the Initial Training Network DAGAL under REA grant agreement number 289313. J.M.G. acknowledges support from the FCT through the Fellowship SFRH/BPD/66958/2009 and the research grant PTDC/FIS-AST/3214/2012. R.G.D. and R.G.B. acknowledge support from the Spanish Ministerio de Economía y Competitividad, through projects AYA2010-15081. R.A.M. is funded by the Spanish program of International Campus of Excellence Moncloa (CEI). This study makes use of the data provided by the Calar Alto Legacy Integral Field Area (CALIFA) survey (http://www. caha.es/CALIFA/). CALIFA is the first legacy survey being performed at Calar Alto. The CALIFA collaboration would like to thank the IAA-CSIC and MPIA-MPG as major partners of the observatory, and CAHA itself, for the unique access to telescope time and support in manpower and infrastructures. The CALIFA collaboration also thanks also the CAHA staff for the dedication to this project. Based on observations collected at the Centro Astronómico Hispano Alemán (CAHA) at Calar Alto, operated jointly by the Max-Planck Institut für Astronomie and the Instituto de Astrofísica de Andalucía (CSIC). The STARLIGHT project is supported by the Brazilian agencies CNPq, CAPES and FAPESP and by the France-Brazil CAPES/Cofecub program. This research has made use of the Asiago Supernova Catalog, the SIMBAD database, operated at CDS, Strasbourg, France, the NASA/IPAC Extragalactic Database (NED), which is operated by the Jet Propulsion Laboratory, California Institute of Technology, under contract with the National Aeronautics and Space Administration, IAU Circulars presented by the Central Bureau for Astronomical Telegrams, and data products from SDSS and SDSS-II surveys.

\section{References}

Abazajian, K. N., Adelman-McCarthy, J. K., Agüeros, M. A., et al. 2009, ApJS, 182,543

Albrecht, A., Bernstein, G., Cahn, R., et al. 2006 [arXiv: astro-ph/0609591] Anderson, J. P., \& James, P. A. 2008, MNRAS, 390, 1527

Anderson, J. P., Covarrubias, R. A., James, P. A., Hamuy, M., \& Habergham, S. M. 2010, MNRAS, 407, 2660

Anderson, J. P., Habergham, S. M., James, P. A., \& Hamuy, M. 2012, MNRAS, 424, 1372

Arcavi, I., Gal-Yam, A., Kasliwal, M. M., et al. 2010, ApJ, 721, 777

Arnett, W. D., Bahcall, J. N., Kirshner, R. P., \& Woosley, S. E. 1989, ARA\&A, 27, 629

Asari, N. V., Cid Fernandes, R., Stasińska, G., et al. 2007, MNRAS, 381, 263

Baldwin, J. A., Phillips, M. M., \& Terlevich, R. 1981, PASP, 93, 5

Barbon, R., Cappellaro, E., \& Turatto, M. 1989, A\&AS, 81, 421

Becker, S. A., \& Iben, Jr., I. 1980, ApJ, 237, 111

Bersten, M. C., Benvenuto, O. G., Nomoto, K., et al. 2012, ApJ, 757, 31

Bersten, M. C., Benvenuto, O. G., Folatelli, G., et al. 2014, AJ, 148, 68

Bertin, E., \& Arnouts, S. 1996, A\&AS, 117, 393

Bethe, H. A., Brown, G. E., Applegate, J., \& Lattimer, J. M. 1979, Nucl. Phys. A, 324, 487

Boissier, S., \& Prantzos, N. 2009, A\&A, 503, 137 
Brandt, T. D., Tojeiro, R., Aubourg, É., et al. 2010, AJ, 140, 804

Bravo, E., Domínguez, I., Badenes, C., Piersanti, L., \& Straniero, O. 2010, ApJ, 711, L66

Brinchmann, J., Charlot, S., White, S. D. M., et al. 2004, MNRAS, 351, 1151

Bruzual, G. 2007, in From Stars to Galaxies: Building the Pieces to Build Up the Universe, eds. A. Vallenari, R. Tantalo, L. Portinari, \& A. Moretti, ASP Conf. Ser., 374, 303

Bruzual, G., \& Charlot, S. 2003, MNRAS, 344, 1000

Cao, Y., Kasliwal, M. M., Arcavi, I., et al. 2013, ApJ, 775, L7

Cappellari, M., \& Copin, Y. 2003, MNRAS, 342, 345

Cardelli, J. A., Clayton, G. C., \& Mathis, J. S. 1989, ApJ, 345, 245

Chabrier, G. 2003, PASP, 115, 763

Childress, M., Aldering, G., Antilogus, P., et al. 2013, ApJ, 770, 107

Cid Fernandes, R., Mateus, A., Sodré, L., Stasińska, G., \& Gomes, J. M. 2005, MNRAS, 358, 363

Cid Fernandes, R., Schoenell, W., Gomes, J. M., et al. 2009, in Rev. Mex. Astron. Astrofis. Conf. Ser., 35, 127

Cid Fernandes, R., Stasińska, G., Schlickmann, M. S., et al. 2010, MNRAS, 403, 1036

Cid Fernandes, R., Pérez, E., García Benito, R., et al. 2013, A\&A, 557, A86

Cid Fernandes, R., González Delgado, R. M., García Benito, R., et al. 2014, A\&A, 561, A130

Crowther, P. A. 2007, ARA\&A, 45, 177

Crowther, P. A. 2013, MNRAS, 428, 1927

Diehl, S., \& Statler, T. S. 2006, MNRAS, 368, 497

Dominguez, I., Chieffi, A., Limongi, M., \& Straniero, O. 1999, ApJ, 524, 226

Domínguez, I., Höflich, P., \& Straniero, O. 2001, ApJ, 557, 279

Elbaz, D., Daddi, E., Le Borgne, D., et al. 2007, A\&A, 468, 33

Eldridge, J. J., Langer, N., \& Tout, C. A. 2011, MNRAS, 414, 3501

Foley, R. J., \& Mandel, K. 2013, ApJ, 778, 167

Fox, O., Skrutskie, M. F., Chevalier, R. A., et al. 2009, ApJ, 691, 650

Gal-Yam, A., Arcavi, I., Ofek, E. O., et al. 2014, Nature, 509, 471

Galbany, L., Miquel, R., Östman, L., et al. 2012, ApJ, 755, 125

Gallagher, J. S., Garnavich, P. M., Berlind, P., et al. 2005, ApJ, 634, 210

Gallagher, J. S., Garnavich, P. M., Caldwell, N., et al. 2008, ApJ, 685, 752

Gaskell, C. M., Cappellaro, E., Dinerstein, H. L., et al. 1986, ApJ, 306, L77

Gogarten, S. M., Dalcanton, J. J., Williams, B. F., et al. 2009, ApJ, 691, 115

González Delgado, R. M., \& Pérez, E. 1997, ApJS, 108, 199

González Delgado, R. M., Cid Fernandes, R., García-Benito, R., et al. 2014a, ApJ, 791, L16

González Delgado, R. M., Pérez, E., Cid Fernandes, R., et al. 2014b, A\&A, 562, A47

Gupta, R. R., D’Andrea, C. B., Sako, M., et al. 2011, ApJ, 740, 92

Habergham, S. M., Anderson, J. P., James, P. A., \& Lyman, J. D. 2014, MNRAS, 441,2230

Hakobyan, A. A., Petrosian, A. R., McLean, B., et al. 2008, A\&A, 488, 523

Hakobyan, A. A., Mamon, G. A., Petrosian, A. R., Kunth, D., \& Turatto, M. 2009, A\&A, 508, 1259

Hakobyan, A. A., Nazaryan, T. A., Adibekyan, V. Z., et al. 2014, MNRAS, 444, 2428

Hammer, F., Flores, H., Schaerer, D., et al. 2006, A\&A, 454, 103

Hamuy, M., Phillips, M. M., Suntzeff, N. B., et al. 1996, AJ, 112, 2391

Hamuy, M., Trager, S. C., Pinto, P. A., et al. 2000, AJ, 120, 1479

Höflich, P., Wheeler, J. C., \& Thielemann, F. K. 1998, ApJ, 495, 617

Howell, D. A., Sullivan, M., Brown, E. F., et al. 2009, ApJ, 691, 661

Hoyle, F., \& Fowler, W. A. 1960, ApJ, 132, 565

Husemann, B., Jahnke, K., Sánchez, S. F., et al. 2013, A\&A, 549, A87

Iben, I., \& Tutukov, A. V. 1984, ApJS, 54, 335

Ivanov, V. D., Hamuy, M., \& Pinto, P. A. 2000, ApJ, 542, 588

James, P. A., \& Anderson, J. P. 2006, A\&A, 453, 57

Jester, S., Schneider, D. P., Richards, G. T., et al. 2005, AJ, 130, 873

Kangas, T., Mattila, S., Kankare, E., et al. 2013, MNRAS, 436, 3464

Kasen, D., Röpke, F. K., \& Woosley, S. E. 2009, Nature, 460, 869

Kauffmann, G., Heckman, T. M., Tremonti, C., et al. 2003, MNRAS, 346, 1055

Kehrig, C., Monreal-Ibero, A., Papaderos, P., et al. 2012, A\&A, 540, A11

Kelly, P. L., \& Kirshner, R. P. 2012, ApJ, 759, 107

Kelly, P. L., Hicken, M., Burke, D. L., Mandel, K. S., \& Kirshner, R. P. 2010, ApJ, 715, 743

Kelz, A., Verheijen, M. A. W., Roth, M. M., et al. 2006, PASP, 118, 129

Kennicutt, Jr., R. C. 1998a, ARA\&A, 36, 189

Kennicutt, Jr., R. C. 1998b, ApJ, 498, 541

Kennicutt, Jr., R. C., Tamblyn, P., \& Congdon, C. E. 1994, ApJ, 435, 22

Kewley, L. J., Dopita, M. A., Sutherland, R. S., Heisler, C. A., \& Trevena, J. 2001, ApJ, 556, 121

Kochanek, C. S., Szczygieł, D. M., \& Stanek, K. Z. 2012, ApJ, 758, 142

Krajnović, D., Cappellari, M., de Zeeuw, P. T., \& Copin, Y. 2006, MNRAS, 366, 787

Kuncarayakti, H., Doi, M., Aldering, G., et al. 2013a, AJ, 146, 30
Kuncarayakti, H., Doi, M., Aldering, G., et al. 2013b, AJ, 146, 31

Lampeitl, H., Smith, M., Nichol, R. C., et al. 2010, ApJ, 722, 566

Leloudas, G., Gallazzi, A., Sollerman, J., et al. 2011, A\&A, 530, A95

Li, W., Leaman, J., Chornock, R., et al. 2011, MNRAS, 412, 1441

López, L. A., Krumholz, M. R., Bolatto, A. D., Prochaska, J. X., \& Ramirez-Ruiz, E. 2011, ApJ, 731, 91

Mannucci, F., della Valle, M., Panagia, N., et al. 2005, A\&A, 433, 807

Maoz, D., \& Badenes, C. 2010, MNRAS, 407, 1314

Maoz, D., Sharon, K., \& Gal-Yam, A. 2010, ApJ, 722, 1879

Maoz, D., Mannucci, F., Li, W., et al. 2011, MNRAS, 412, 1508

Maoz, D., Mannucci, F., \& Brandt, T. D. 2012, MNRAS, 426, 3282

Maoz, D., Mannucci, F., \& Nelemans, G. 2014, ARA\&A, 52, 107

Marigo, P., \& Girardi, L. 2007, A\&A, 469, 239

Marigo, P., Girardi, L., Bressan, A., et al. 2008, A\&A, 482, 883

Marino, R. A., Gil de Paz, A., Castillo-Morales, A., et al. 2012, ApJ, 754, 61

Mármol-Queraltó, E., Sánchez, S. F., Marino, R. A., et al. 2011, A\&A, 534, A8

Mast, D., Rosales-Ortega, F. F., Sánchez, S. F., et al. 2014, A\&A, 561, A129

Masters, K. L., Mosleh, M., Romer, A. K., et al. 2010, MNRAS, 405, 783

Mateus, A., Sodré, L., Cid Fernandes, R., et al. 2006, MNRAS, 370, 721

Matteucci, F. 2012, Chemical Evolution of Galaxies (Springer)

Mazzali, P. A., Röpke, F. K., Benetti, S., \& Hillebrandt, W. 2007, Science, 315, 825

Modjaz, M., Kewley, L., Kirshner, R. P., et al. 2008, AJ, 135, 1136

Modjaz, M., Kewley, L., Bloom, J. S., et al. 2011, ApJ, 731, L4

Modjaz, M., Blondin, S., Kirshner, R. P., et al. 2014, AJ, 147, 99

Neill, J. D., Sullivan, M., Howell, D. A., et al. 2009, ApJ, 707, 1449

Nomoto, K., Iwamoto, K., Suzuki, T., et al. 1996, in Compact Stars in Binaries, eds. J. van Paradijs, E. P. J. van den Heuvel, \& E. Kuulkers, IAU Symp., 165, 119

O’Donnell, J. E. 1994, ApJ, 422, 158

Osterbrock, D. E., \& Ferland, G. J. 2006, Astrophysics of gaseous nebulae and active galactic nuclei (Sausalito: University Science Books)

Pagel, B. E. J. 1997, Nucleosynthesis and Chemical Evolution of Galaxies (Cambridge University Press)

Pan, Y.-C., Sullivan, M., Maguire, K., et al. 2014, MNRAS, 438, 1391

Papaderos, P., Gomes, J. M., Vílchez, J. M., et al. 2013, A\&A, 555, L1

Pérez, E., Cid Fernandes, R., González Delgado, R. M., et al. 2013, ApJ, 764, L1

Perlmutter, S., Aldering, G., Goldhaber, G., et al. 1999, ApJ, 517, 565

Phillips, M. M., Lira, P., Suntzeff, N. B., et al. 1999, AJ, 118, 1766

Podsiadlowski, P., Joss, P. C., \& Hsu, J. J. L. 1992, ApJ, 391, 246

Prieto, J. L., Stanek, K. Z., \& Beacom, J. F. 2008, ApJ, 673, 999

Raskin, C., Scannapieco, E., Rhoads, J., \& Della Valle, M. 2009, ApJ, 707, 74

Riess, A. G., Filippenko, A. V., Challis, P., et al. 1998, AJ, 116, 1009

Rigault, M., Copin, Y., Aldering, G., et al. 2013, A\&A, 560, A66

Röpke, F. K., Gieseler, M., Reinecke, M., Travaglio, C., \& Hillebrandt, W. 2006, A\&A, 453, 203

Rosales-Ortega, F. F., Kennicutt, R. C., Sánchez, S. F., et al. 2010, MNRAS, 405, 735

Roth, M. M., Kelz, A., Fechner, T., et al. 2005, PASP, 117, 620

Sánchez, S. F., Kennicutt, R. C., Gil de Paz, A., et al. 2012a, A\&A, 538, A8

Sánchez, S. F., Rosales-Ortega, F. F., Marino, R. A., et al. 2012b, A\&A, 546, A2

Sánchez, S. F., Rosales-Ortega, F. F., Jungwiert, B., et al. 2013, A\&A, 554, A58

Sánchez, S. F., Rosales-Ortega, F. F., Iglesias-Páramo, J., et al. 2014, A\&A, 563, A49

Sánchez-Blázquez, P., Peletier, R. F., Jiménez-Vicente, J., et al. 2006, MNRAS, 371,703

Sánchez-Blázquez, P., Rosales-Ortega, F., Mendez-Abreu, J., et al. 2014, A\&A, 570, A6

Scannapieco, E., \& Bildsten, L. 2005, ApJ, 629, L85

Schlegel, D. J., Finkbeiner, D. P., \& Davis, M. 1998, ApJ, 500, 525

Shao, X., Liang, Y. C., Dennefeld, M., et al. 2014, ApJ, 791, 57

Singh, R., van de Ven, G., Jahnke, K., et al. 2013, A\&A, 558, A43

Smartt, S. J. 2009, ARA\&A, 47, 63

Smith, N., Li, W., Silverman, J. M., Ganeshalingam, M., \& Filippenko, A. V. 2011, MNRAS, 415, 773

Stanishev, V., Rodrigues, M., Mourão, A., \& Flores, H. 2012, A\&A, 545, A58

Stoll, R., Prieto, J. L., Stanek, K. Z., \& Pogge, R. W. 2013, ApJ, 773, 12

Suh, H., Yoon, S.-C., Jeong, H., \& Yi, S. K. 2011, ApJ, 730, 110

Sullivan, M., Le Borgne, D., Pritchet, C. J., et al. 2006, ApJ, 648, 868

Sullivan, M., Conley, A., Howell, D. A., et al. 2010, MNRAS, 406, 782

Timmes, F. X., Brown, E. F., \& Truran, J. W. 2003, ApJ, 590, L83

Umeda, H., Nomoto, K., Kobayashi, C., Hachisu, I., \& Kato, M. 1999, ApJ, 522, L43

Veilleux, S., \& Osterbrock, D. E. 1987, ApJS, 63, 295

Verheijen, M. A. W., Bershady, M. A., Andersen, D. R., et al. 2004, Astron. Nachr., 325, 151

Walcher, C. J., Wisotzki, L., Bekeraité, S., et al. 2014, A\&A, 569, A1 
L. Galbany et al.: Nearby supernova host galaxies from the CALIFA Survey. I.

Wang, J., Deng, J. S., \& Wei, J. Y. 2010, MNRAS, 405, 2529

Whelan, J., \& Iben, I. J. 1973, ApJ, 186, 1007

1 CENTRA - Centro Multidisciplinar de Astrofísica, Instituto Superior Técnico, Av. Rovisco Pais 1, 1049-001 Lisbon, Portugal

2 Millennium Institute of Astrophysics, Universidad de Chile, Casilla 36-D, Santiago, Chile

3 Departamento de Astronomía, Universidad de Chile, Casilla 36-D, Santiago, Chile

4 European Southern Observatory, Alonso de Cordova 3107 Casilla 19001 - Vitacura-Santiago, Chile

5 GEPI, Observatoire de Paris, UMR 8111, CNRS, Université Paris Diderot, 5 place Jules Janssen, 92190 Meudon, France

6 Instituto de Astrofśica de Andalucía (CSIC), Glorieta de la Astronomía s/n, Aptdo. 3004, 18080-Granada, Spain

7 Instituto de Cosmologia, Relatividade e Astrofísica - ICRA, Centro Brasileiro de Pesquisas Físicas, Rua Dr. Xavier Sigaud 150, CEP 22290-180, Rio de Janeiro, RJ, Brazil

8 Instituto de Astronomía, Universidad Nacional Autónoma de México, A.P. 70-264, 04510, Mexico, D.F

9 Department of Physics and Astronomy, University of Pittsburgh, Allen Hall, 3941 O'Hara St, Pittsburgh, PA 15260, USA

10 Instituto de Astrofísica de Canarias (IAC), 38205 La Laguna, Tenerife, Spain

11 Departamento de Astrofísica, Universidad de La Laguna, 38205 La Laguna, Tenerife, Spain
12 Sydney Institute for Astronomy, School of Physics A28, University of Sydney, NSW 2006, Australia

13 Centro de Astrofísica and Faculdade de Ciencias, Universidade do Porto, Rua das Estrelas, 4150-762 Porto, Portugal

14 Max-Planck-Institut für Astronomie, Konigstuhl 17, 69117 Heidelberg, Germany

15 Australian Astronomical Observatory, PO Box 915, North Ryde, NSW 1670, Australia

16 Department of Physics and Astronomy, Macquarie University, NSW 2109, Australia

17 School of Physics and Astronomy, University of St Andrews, North Haugh, St Andrews, KY16 9SS, UK (SUPA)

18 CEI Campus Moncloa, UCM-UPM, Departamento de Astrofísica y CC. de la Atmósfera, Facultad de CC. Físicas, Universidad Complutense de Madrid, Avda.Complutense s/n, 28040 Madrid, Spain

19 Departamento de Investigación Básica, CIEMAT, Avda. Complutense 40, 28040 Madrid, Spain

${ }^{20}$ Centro de Estudios de la Física del Cosmos de Aragón, 44001 Teruel, Spain

21 Visiting Scientist: Departamento de Física Teorica, Facultad de Ciencias, Universidad de Zaragoza, Spain

22 Instituto Nacional de Astrofísica, Óptica y Electrónica, Luis E. Erro 1, 72840 Tonantzintla, Puebla, Mexico 
Table 2. Properties of the 50 SN host galaxies observed by the CALIFA Survey and the 58 SNe used in this study.

\begin{tabular}{|c|c|c|c|c|c|c|c|c|c|c|}
\hline Galaxy & Morphology & $z$ & $E(B-V)$ & $\begin{array}{c}\text { PA } \\
{[\mathrm{deg}]}\end{array}$ & $b / a$ & $S N$ & Type & $\begin{array}{l}\text { RA offset } \\
\text { [arcsec] }\end{array}$ & $\begin{array}{c}\text { Dec offset } \\
\text { [arcsec] }\end{array}$ & $\begin{array}{c}\text { Separation } \\
\text { [arcsec] }\end{array}$ \\
\hline \multirow[t]{2}{*}{ UGC 00005} & \multirow[t]{2}{*}{ SABbc } & \multirow[t]{2}{*}{0.024253} & \multirow[t]{2}{*}{0.041} & \multirow[t]{2}{*}{140.1} & \multirow[t]{2}{*}{0.55} & $2000 \mathrm{da}$ & II & +12.8 & +9.3 & 15.1 \\
\hline & & & & & & $20031 \mathrm{q}$ & Ia & -24.5 & +5.6 & 25.0 \\
\hline UGC $00139^{\dagger}$ & $\mathrm{SAB}(\mathrm{s}) \mathrm{c} ?$ & 0.013219 & 0.043 & 169.5 & 0.54 & 1998dk & Ia & +5.4 & +3.1 & 6.4 \\
\hline UGC 00148 & S? & 0.014053 & 0.062 & 7.9 & 0.40 & 2003ld & II & +8.6 & -1.3 & 8.2 \\
\hline NGC 0214 & $\mathrm{SAB}(\mathrm{r}) \mathrm{c}$ & 0.015134 & 0.035 & 144.7 & 0.66 & $2005 \mathrm{db}$ & IIn & -16.0 & -2.0 & 17.9 \\
\hline NGC 0523 & pec & 0.015871 & 0.054 & 179.2 & 0.40 & 2001en & Ia & +25.8 & $+0.1^{\square}$ & 26.6 \\
\hline NGC 0774 & S0 & 0.015411 & 0.061 & 75.4 & 0.62 & 2006ee & II & +9.5 & -9.4 & 13.9 \\
\hline NGC 0776^ & $\mathrm{SAB}(\mathrm{rs}) \mathrm{b}$ & 0.016415 & 0.097 & 65.2 & 0.85 & 1999di & $\mathrm{Ib}$ & +5.2 & -17.0 & 17.8 \\
\hline NGC $0932^{\dagger}$ & $\mathrm{SAa}$ & 0.013603 & 0.134 & 172.9 & 0.94 & $1992 b f$ & I & +9.4 & -10.8 & 13.3 \\
\hline NGC $1056^{\star}$ & $\mathrm{Sa} ?$ & 0.005154 & 0.149 & 69.7 & 0.54 & $2011 \mathrm{aq}$ & II & -2.9 & -0.9 & 2.5 \\
\hline NGC $1060^{\dagger}$ & SO-? & 0.017312 & 0.172 & 0.1 & 0.83 & $2004 \mathrm{fd}$ & $\mathrm{Ia}$ & +2.5 & -4.3 & 5.0 \\
\hline NGC 1093 & SABab? & 0.017646 & 0.087 & 9.1 & 0.61 & 2009ie & IIP & -27.3 & -19.2 & 33.4 \\
\hline UGC 03151 & $\mathrm{~S}$ & 0.014600 & 0.496 & 7.0 & 0.38 & 1995bd & Ia-pec & +23.6 & -1.6 & 23.6 \\
\hline NGC 2347 & $\left(\mathrm{R}^{\prime}\right) \mathrm{SA}(\mathrm{r}) \mathrm{b}$ ? & 0.014747 & 0.079 & 99.7 & 0.66 & 2001ee & II & -5.0 & -20.0 & 12.7 \\
\hline \multirow[t]{2}{*}{ UGC 04132} & \multirow[t]{2}{*}{ Sbc } & \multirow[t]{2}{*}{0.017409} & \multirow[t]{2}{*}{0.067} & \multirow[t]{2}{*}{116.7} & \multirow[t]{2}{*}{0.42} & $2005 \mathrm{en}$ & II & -5.8 & +6.9 & 9.5 \\
\hline & & & & & & 2005 eo & Ic & +11.0 & +26.1 & 29.8 \\
\hline NGC 2623 & pec & 0.018509 & 0.041 & 118.4 & 0.64 & $1999 \mathrm{gd}$ & Ia & +7.3 & +17.4 & 17.9 \\
\hline NGC 2906 & Scd? & 0.007138 & 0.047 & 170.7 & 0.51 & 2005ip & $\mathrm{IIn}^{*}$ & +2.8 & +14.2 & 14.3 \\
\hline NGC 3057 & $\mathrm{SB}(\mathrm{s}) \mathrm{dm}$ & 0.005084 & 0.023 & 90.0 & 0.68 & $1997 \mathrm{cx}$ & II & -5.0 & +15.0 & 19.7 \\
\hline NGC 3687 & $\left(\mathrm{R}^{\prime}\right) \mathrm{SAB}(\mathrm{r}) \mathrm{bc}$ ? & 0.008362 & 0.022 & 57.0 & 0.98 & 1989A & Ia & -21.0 & -18.0 & 33.6 \\
\hline \multirow[t]{2}{*}{ NGC 3811} & \multirow[t]{2}{*}{$\mathrm{SB}(\mathrm{r}) \mathrm{cd} ?$} & 0.010357 & 0.019 & 87.9 & 0.93 & $1969 \mathrm{C}$ & Ia & +9.0 & +6.0 & 33.6 \\
\hline & & & & & & $1971 K$ & IIP & -30.0 & -17.0 & 35.9 \\
\hline NGC $4210^{\star}$ & $\mathrm{SB}(\mathrm{r}) \mathrm{b}$ & 0.009113 & 0.018 & 7.4 & 0.79 & 2002ho & Ic & +12.9 & -12.2 & 17.8 \\
\hline NGC 4644 & $\mathrm{SBb} ?$ & 0.016501 & 0.020 & 145.1 & 0.45 & $2007 \mathrm{~cm}$ & IIn & +21.5 & +13.5 & 25.4 \\
\hline NGC 4874 & cD0 & 0.023937 & 0.009 & 90.5 & 0.92 & $1981 G$ & Ia & +15.0 & +10.0 & 12.1 \\
\hline NGC 4961 & $\mathrm{SB}(\mathrm{s}) \mathrm{cd}$ & 0.008456 & 0.011 & 14.2 & 0.68 & $2005 \mathrm{az}$ & Ic & -8.0 & +5.5 & 9.7 \\
\hline NGC 5000^ & $\mathrm{SB}(\mathrm{rs}) \mathrm{bc}$ & 0.018706 & 0.009 & 92.2 & 0.98 & $2003 \mathrm{el}$ & Ic & -16.7 & -2.7 & 17.2 \\
\hline UGC 08250^ & Scd? & 0.017646 & 0.015 & 101.0 & 0.26 & $2013 \mathrm{~T}$ & Ia & -5.8 & -33.8 & 34.5 \\
\hline NGC 5056 & Scd? & 0.018653 & 0.012 & 85.6 & 0.66 & $2005 \mathrm{au}$ & II & +1.0 & -21.0 & 20.7 \\
\hline NGC $5157^{\dagger}$ & $\mathrm{SAB}(\mathrm{r}) \mathrm{a}$ & 0.024424 & 0.013 & 174.2 & 0.94 & $1995 \mathrm{~L}$ & Ia & +18.9 & +2.0 & 22.5 \\
\hline NGC $5378^{\star}$ & $\left(\mathrm{R}^{\prime}\right) \mathrm{SB}(\mathrm{r}) \mathrm{a}$ & 0.010147 & 0.013 & 9.5 & 0.78 & 1991ak & Ia & -28.0 & -19.0 & 34.1 \\
\hline NGC $5421^{\dagger}$ & $\mathrm{P}$ & 0.026315 & 0.015 & 82.4 & 0.81 & $2012 \mathrm{~T}$ & Ia-pec & +3.5 & -3.4 & 17.3 \\
\hline NGC $5480^{\dagger}$ & $\mathrm{SA}(\mathrm{s}) \mathrm{c} ?$ & 0.006191 & 0.019 & 89.6 & 0.82 & $1988 \mathrm{~L}$ & $\mathrm{Ib}$ & +3.0 & +14.0 & 10.7 \\
\hline NGC $5611^{\dagger}$ & So & 0.006721 & 0.012 & 149.7 & 0.53 & 2012ei & Ia & +14.1 & +5.8 & 13.1 \\
\hline NGC 5630 & Sdm: & 0.008856 & 0.011 & 178.2 & 0.52 & 2006am & IIn & +7.5 & +6.8 & 10.3 \\
\hline & & & & & & $2005 \mathrm{dp}$ & II & -1.0 & -14.0 & 12.9 \\
\hline NGC 5682* & $\mathrm{SB}(\mathrm{s}) \mathrm{b}$ & 0.007581 & 0.033 & 37.7 & 0.40 & $2005 \mathrm{ci}$ & II & -0.6 & +6.1 & 7.0 \\
\hline NGC 5714 & Scd? & 0.007462 & 0.015 & 172.9 & 0.26 & $2003 \mathrm{dr}$ & $\mathrm{Ib} / \mathrm{c}-\mathrm{pec}$ & -3.7 & -13.9 & 14.9 \\
\hline NGC $5735^{\dagger}$ & $\mathrm{SB}(\mathrm{rs}) \mathrm{bc}$ & 0.012482 & 0.017 & 135.4 & 0.99 & $2006 q p$ & IIb & -35.0 & -10.0 & 35.3 \\
\hline NGC $5772^{\dagger}$ & SA(r)b? & 0.016345 & 0.018 & 125.7 & 0.55 & 2002ee & IIP & +18.3 & +33.9 & 44.6 \\
\hline NGC 5829 & $\mathrm{SA}(\mathrm{s}) \mathrm{c}$ & 0.018797 & 0.044 & 169.7 & 0.96 & 2008B & IIn & +23.0 & +7.0 & 23.6 \\
\hline NGC 5888 & $\mathrm{SB}(\mathrm{s}) \mathrm{bc}$ & 0.029123 & 0.023 & 59.5 & 0.61 & 2007Q & II & +14.5 & -13.8 & 14.8 \\
\hline & & & & & & $2010 \mathrm{fv}$ & II & -9.4 & +12.7 & 16.0 \\
\hline UGC $09842^{\dagger}$ & $\mathrm{SBb}$ & 0.029726 & 0.014 & 160.9 & 0.46 & 2012as & IIn & +30.9 & +12.9 & 34.6 \\
\hline NGC 5980 & S & 0.013649 & 0.035 & 103.9 & 0.43 & $2004 \mathrm{ci}$ & II & -10.1 & -2.3 & 8.6 \\
\hline UGC 10097 & So & 0.019887 & 0.018 & 45.4 & 0.44 & 2004di & Ia & -23.0 & -11.0 & 26.0 \\
\hline UGC $10331^{\star}$ & S pec & 0.014914 & 0.013 & 49.5 & 0.39 & 2011jg & IIb & -15.0 & +17.0 & 24.5 \\
\hline NGC $6146^{\star}$ & $\mathrm{E}$ ? & 0.029420 & 0.009 & 170.0 & 0.99 & $2009 \mathrm{fl}$ & Ia & -2.3 & -13.2 & 13.8 \\
\hline NGC 6166 & cD2 pec & 0.030354 & 0.012 & 123.8 & 0.95 & $2009 \mathrm{eu}$ & Ia & +32.7 & +7.8 & 31.4 \\
\hline NGC $6173^{\star}$ & $\mathrm{E}$ & 0.029300 & 0.007 & 53.1 & 0.77 & $2009 \mathrm{fv}$ & Ia & -7.8 & -0.4 & 7.7 \\
\hline NGC 6186 & $\left(\mathrm{R}^{\prime}\right) \mathrm{SB}(\mathrm{s}) \mathrm{a}$ & 0.009797 & 0.047 & 154.0 & 0.68 & 2011gd & $\mathrm{Ib}$ & +2.9 & +0.9 & 2.9 \\
\hline MCG -01-54-016 & S? & 0.009773 & 0.058 & 120.6 & 0.23 & 2001ch & Ic & -12.0 & -14.0 & 14.6 \\
\hline NGC 7311 & Sab & 0.015120 & 0.131 & 103.2 & 0.48 & $2005 \mathrm{kc}$ & Ia & +7.6 & -7.4 & 10.8 \\
\hline NGC $7321^{\star}$ & $\mathrm{SB}(\mathrm{r}) \mathrm{b}$ & 0.023833 & 0.046 & 99.8 & 0.74 & 2008gj & Ic & +7.6 & +37.0 & 37.6 \\
\hline & & & & & & 2013di & Ia & -7.8 & -24.2 & 25.4 \\
\hline NGC 7364 & S0/a pec & 0.016228 & 0.060 & 157.8 & 0.77 & 2009fk & Ia & -6.7 & +1.5 & 7.2 \\
\hline & & & & & & 2006lc & $\mathrm{Ib}^{*}$ & +1.4 & -10.0 & 10.1 \\
\hline & & & & & & $2011 \mathrm{im}$ & Ia & +13.0 & -18.8 & 22.4 \\
\hline
\end{tabular}

Notes. The morphological galaxy type, redshift, Milky Way dust reddening, and SN angular separation are taken from the NED database. SN type and offset (positive in the $\mathrm{N}$ and $\mathrm{E}$ direction) obtained from the Asiago SN catalogue. The position angle (PA, W to N) and the axis ratio $(b / a)$ are calculated in this work. ${ }^{(\star)}$ Publicly available in CALIFA DR1. ${ }^{(\dagger)}$ Only observed with the V500 grating. ${ }^{(*)}$ SN classification changed from Asiago SN catalogue: 2005ip from II to IIn in Fox et al. (2009); 2006lc from Ib/c to Ib following Leloudas et al. (2011). 
L. Galbany et al.: Nearby supernova host galaxies from the CALIFA Survey. I.

Table 3. Properties of the 31 SN host galaxies used in this study not observed by the CALIFA Survey: 4 from the feasibility study for CALIFA, 8 from the PINGS Survey, NGC 5668 and NGC 3982 from Marino et al. (2012) and Marino et al. (in prep.), 5 from S12, and 12 from CALIFAextensions.

\begin{tabular}{|c|c|c|c|c|c|c|c|c|c|c|}
\hline Galaxy & Morphology & $z$ & $E(B-V)$ & $\begin{array}{c}\text { PA } \\
{[\mathrm{deg}]}\end{array}$ & $b / a$ & $S N$ & Type & $\begin{array}{l}\text { RA offset } \\
\text { [arcsec] }\end{array}$ & $\begin{array}{c}\text { Dec offset } \\
\text { [arcsec] }\end{array}$ & $\begin{array}{c}\text { Separation } \\
\text { [arcsec] }\end{array}$ \\
\hline UGC 01087 & $\mathrm{SA}(\mathrm{rs}) \mathrm{c}$ & 0.014960 & 0.054 & 6.5 & 0.98 & $1999 \mathrm{dk}$ & Ia & +4.1 & +26.2 & 27.1 \\
\hline UGC 04036 & $\mathrm{SAB}(\mathrm{r}) \mathrm{b} ?$ & 0.011575 & 0.027 & 67.6 & 0.97 & $1995 \mathrm{E}$ & Ia & +7.0 & -22.0 & 23.5 \\
\hline UGC 04107 & $\mathrm{SA}(\mathrm{rs}) \mathrm{c}$ & 0.011688 & 0.042 & 161.0 & 0.97 & $1997 \mathrm{ef}$ & $\mathrm{Ic}^{*}$ & +10.0 & -20.0 & 24.1 \\
\hline UGC 05100 & $\mathrm{SB}(\mathrm{s}) \mathrm{b}$ & 0.018393 & 0.038 & 44.0 & 0.88 & $2002 \mathrm{au}$ & $\mathrm{IIb}^{*}$ & -16.0 & -13.6 & 20.6 \\
\hline \multirow[t]{2}{*}{ NGC $0628^{i i}$} & $\mathrm{SA}(\mathrm{s}) \mathrm{c}$ & 0.002192 & 0.070 & 97.4 & 0.98 & 2003gd & IIP & +13.2 & -161.0 & 161.8 \\
\hline & & & & & & 2013еj & IIP & +92.0 & -135.0 & 163.4 \\
\hline \multirow[t]{2}{*}{ NGC 1058} & $\mathrm{SA}(\mathrm{rs}) \mathrm{c}$ & 0.001728 & 0.062 & 158.0 & 0.99 & $2007 \mathrm{gr}$ & Ic & -24.8 & +15.8 & 28.9 \\
\hline & & & & & & $1961 \mathrm{~V}$ & II-pec ${ }^{i}$ & +76.0 & +17.0 & 78.4 \\
\hline NGC 1637 & $\mathrm{SAB}(\mathrm{rs}) \mathrm{c}$ & 0.002392 & 0.040 & 123.3 & 0.89 & 1999em & IIP & -15.4 & -17.0 & 23.0 \\
\hline NGC $3184^{i i}$ & $\mathrm{SAB}(\mathrm{rs}) \mathrm{cd}$ & 0.001975 & 0.022 & 71.1 & 0.96 & 1999gi & IIP & -3.5 & +60.5 & 62.5 \\
\hline NGC 3310 & $\mathrm{SAB}(\mathrm{r}) \mathrm{bc}$ pec & 0.003312 & 0.023 & 69.8 & 0.78 & $1991 N$ & Ic & +5.0 & -7.0 & 8.5 \\
\hline \multirow[t]{2}{*}{ NGC 6643} & $\mathrm{SA}(\mathrm{rs}) \mathrm{c}$ & 0.004950 & 0.060 & 118.0 & 0.87 & $2008 \mathrm{ij}$ & II & +23.0 & -11.0 & 24.3 \\
\hline & & & & & & 2008bo & IIb & +31.0 & +15.0 & 34.9 \\
\hline NGC $7319^{i i}$ & $\mathrm{SB}(\mathrm{s}) \mathrm{bc}$ pec & 0.022507 & 0.078 & 57.5 & 0.89 & $1971 \mathrm{P}$ & I & +27.0 & -18.0 & 32.9 \\
\hline NGC 7771 & $\mathrm{SB}(\mathrm{s}) \mathrm{a}$ & 0.014267 & 0.074 & 160.5 & 0.43 & $2003 \mathrm{hg}$ & II & -11.5 & -3.9 & 10.3 \\
\hline NGC $3982^{i i}$ & $\mathrm{SAB}(\mathrm{r}) \mathrm{b} ?$ & 0.003699 & 0.014 & 101.7 & 0.86 & $1998 \mathrm{aq}$ & Ia & -18.0 & +7.0 & 23.0 \\
\hline \multirow{2}{*}{ NGC 5668} & $\mathrm{SA}(\mathrm{s}) \mathrm{d}$ & 0.005260 & 0.037 & 108.5 & 0.92 & 1954B & Ia & -2.0 & -20.0 & 20.3 \\
\hline & & & & & & $2004 \mathrm{G}$ & II & -43.0 & -12.5 & 45.6 \\
\hline \multirow[t]{2}{*}{ NGC $0105^{i i}$} & Sab: & 0.017646 & 0.073 & 78.0 & 0.81 & $2007 \mathrm{~A}$ & Ia & -1.2 & +10.1 & 9.5 \\
\hline & & & & & & $1997 \mathrm{cw}$ & Ia-pec & +7.6 & +4.2 & 18.6 \\
\hline NGC $0976^{i i}$ & SA(rs)c: & 0.014327 & 0.110 & 78.5 & 0.82 & $1999 d q$ & Ia-pec & -4.3 & -6.4 & 7.7 \\
\hline UGC $04008^{i i}$ & $\mathrm{~S} 0 / \mathrm{a}$ & 0.030751 & 0.047 & 74.7 & 0.62 & $2007 R$ & Ia & -1.9 & -3.9 & 30.4 \\
\hline CGCG 207-042 $2^{i i}$ & $\mathrm{SBbc}$ & 0.031592 & 0.046 & 65.1 & 0.62 & 2006te & Ia & -5.5 & -1.7 & 5.6 \\
\hline UGC $05129^{i i}$ & $\mathrm{Sa}$ & 0.013539 & 0.022 & 13.9 & 0.60 & $2001 \mathrm{fe}$ & Ia & -13.5 & -0.1 & 12.2 \\
\hline MCG -02-02-086 & SB0(r) pec? & 0.055672 & 0.037 & 64.0 & 0.59 & 2003 ic & Ia & -2.1 & -7.6 & 8.5 \\
\hline NGC 0495 & $\left(\mathrm{R}^{\prime}\right) \mathrm{SB} 0 / \mathrm{a}(\mathrm{s})$ pec? & 0.013723 & 0.071 & 54.0 & 0.79 & 1999ej & Ia & +17.7 & -20.1 & 26.4 \\
\hline UGC 01635 & $\mathrm{SAbc}$ & 0.011755 & 0.055 & 51.3 & 0.96 & $2003 \mathrm{G}$ & IIn & +6.0 & +9.8 & 11.8 \\
\hline \multirow[t]{2}{*}{ UGC 03555} & $\mathrm{SAB}(\mathrm{rs}) \mathrm{bc}$ & 0.016128 & 0.087 & 135.7 & 0.98 & 2004ge & Ic & +6.2 & -1.4 & 6.4 \\
\hline & & & & & & $1999 \mathrm{ed}$ & II & +17.4 & -9.0 & 19.6 \\
\hline UGC 04455 & $\mathrm{SB}(\mathrm{r}) \mathrm{a}$ & 0.030908 & 0.034 & 107.8 & 0.82 & 2007bd & Ia & +7.6 & +5.1 & 8.6 \\
\hline NGC 2691 & Sa? & 0.013279 & 0.024 & 73.2 & 0.59 & $2011 \mathrm{hr}$ & Ia-pec & -3.4 & -3.7 & 4.7 \\
\hline NGC 3655 & $\mathrm{SA}(\mathrm{s}) \mathrm{c} ?$ & 0.004913 & 0.026 & 118.5 & 0.70 & $2002 \mathrm{ji}$ & $\mathrm{Ib}^{*}$ & -22.4 & -14.0 & 25.4 \\
\hline NGC 3913 & $\left(\mathrm{R}^{\prime}\right) \mathrm{SA}(\mathrm{rs}) \mathrm{d}$ ? & 0.003182 & 0.013 & 72.0 & 0.88 & $1963 \mathrm{~J}$ & Ia & -5.0 & -12.0 & 13.8 \\
\hline NGC 6786 & SB? & 0.025017 & 0.141 & 147.9 & 0.99 & 2004ed & II & -1.8 & -9.8 & 9.1 \\
\hline UGC 11975 & $\mathrm{~S} 0 / \mathrm{a}$ & 0.020914 & 0.117 & 18.6 & 0.84 & $2011 \mathrm{fs}$ & Ia & -2.0 & +34.0 & 33.9 \\
\hline NGC 7253 & S? & 0.014987 & 0.066 & 144.1 & 0.47 & 2002jg & Ia & -20.0 & -13.1 & 20.5 \\
\hline NGC 7469 & SAbc & 0.016317 & 0.069 & 40.4 & 0.83 & $2008 \mathrm{ec}$ & Ia & +13.7 & -7.4 & 15.4 \\
\hline
\end{tabular}

Notes. A total of $37 \mathrm{SNe}$ are collected. Parameters of the sources are detailed in Table 2. ${ }^{(i)} \mathrm{SN} 1961 \mathrm{~V}$ has suspected to be a SN impostor, but was included here following the conclusions of Smith et al. (2011) and Kochanek et al. (2012). ${ }^{(i)}$ The datacubes of these galaxies have a spatial resolution of 2" pixel $^{-1}$. (*) SN classification changed from Asiago SN catalogue: 2002au from Ia to IIb in Li et al. (2011). 1997ef from Ib/c-pec to Ic, and 2002ji from Ib/c to Ib in Modjaz et al. (2014). 


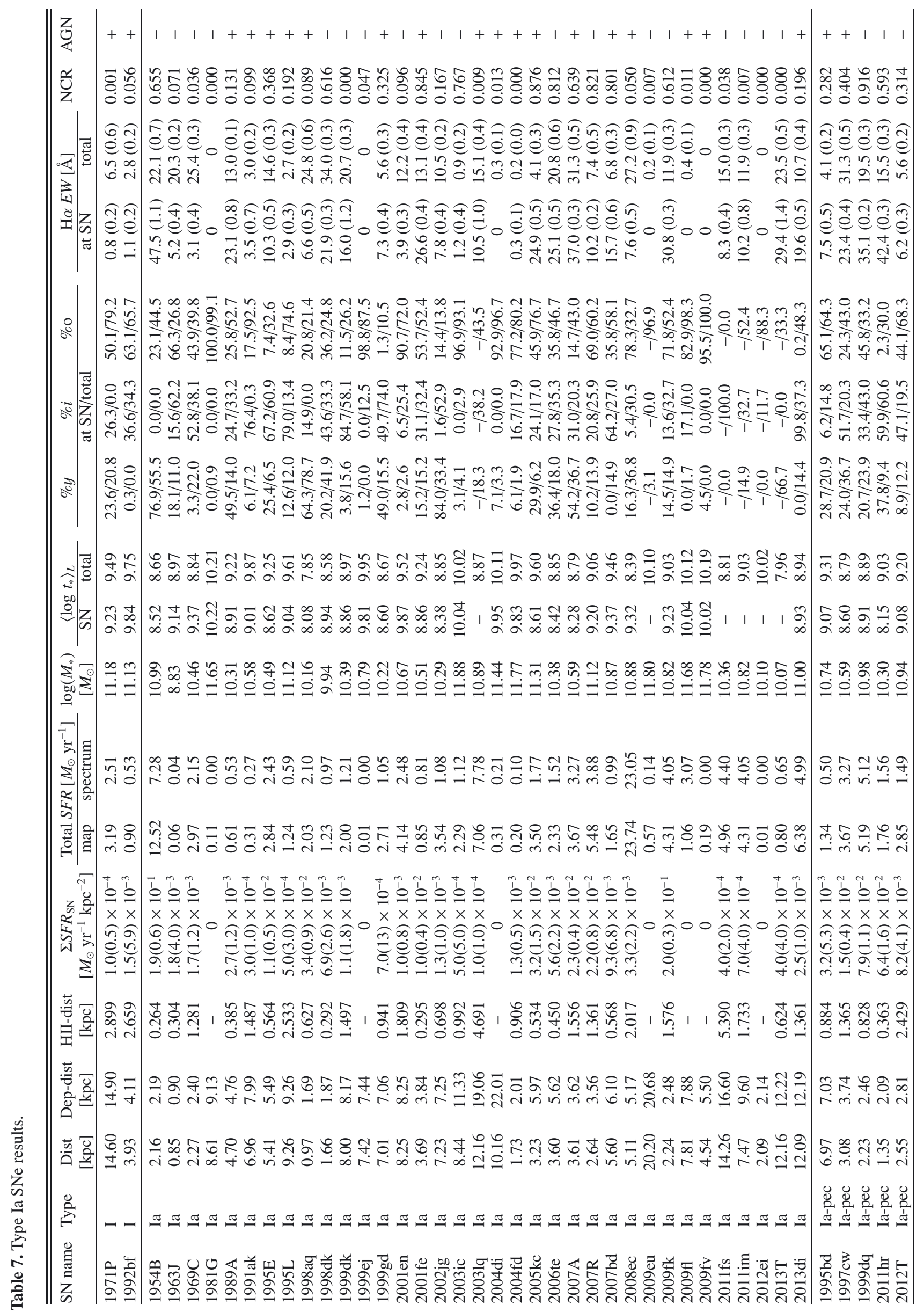


L. Galbany et al.: Nearby supernova host galaxies from the CALIFA Survey. I.

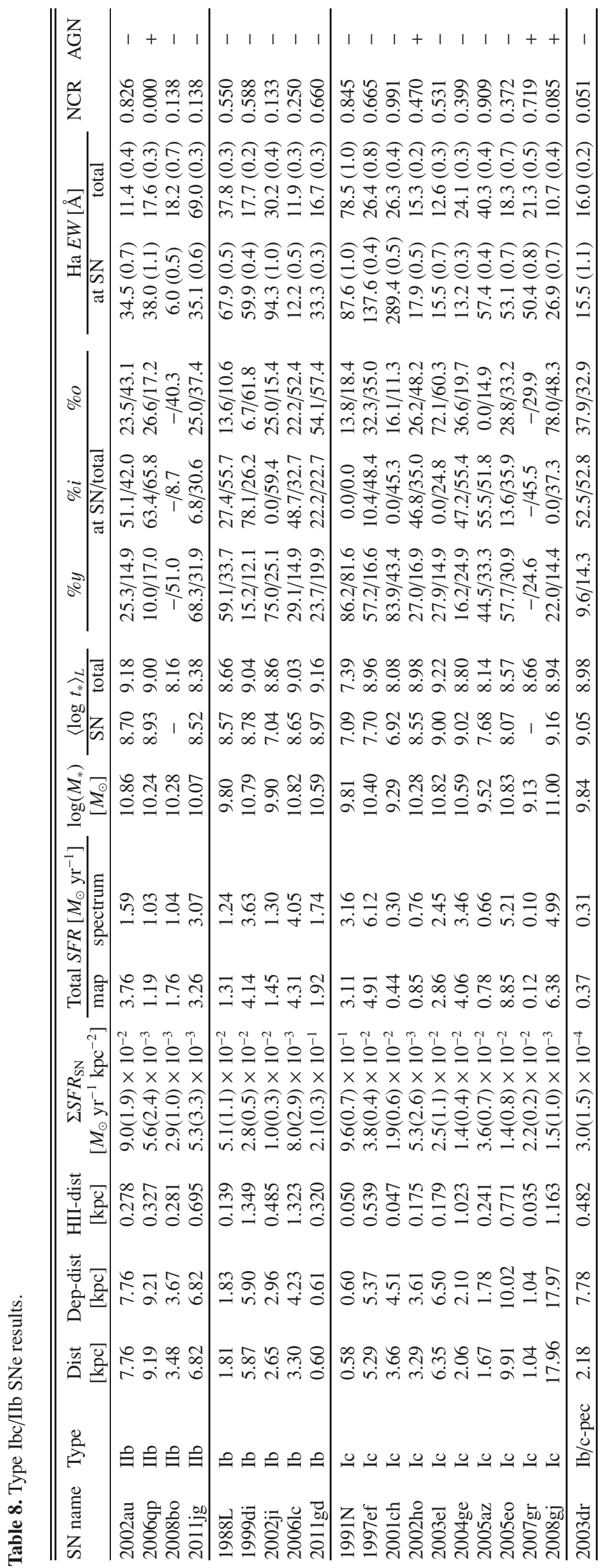




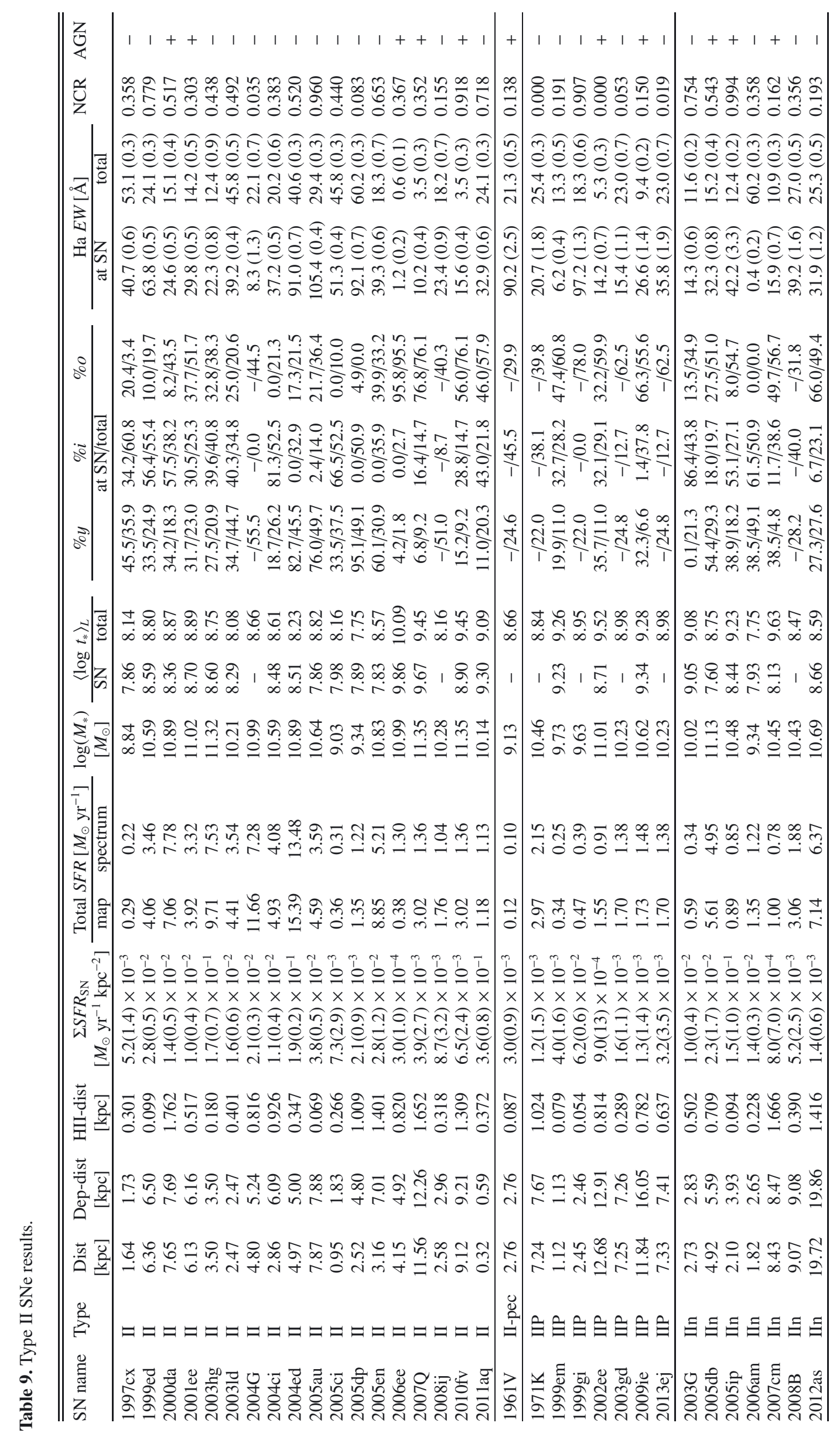

